\title{
On Bifurcating Time-Periodic Flow of a Navier-Stokes Liquid past a Cylinder
}

\author{
Giovanni P. Galdi *
}

\begin{abstract}
We provide general sufficient conditions for branching out of a timeperiodic family of solutions from steady-state solutions to the twodimensional Navier-Stokes equations in the exterior of a cylinder. To this end, we first show that the problem can be formulated as a coupled elliptic-parabolic nonlinear system in appropriate function spaces. This is obtained by separating the time-independent averaged component of the velocity field from its "purely periodic" one. We then prove that time-periodic bifurcation occurs, provided the linearized time-independent operator of the parabolic problem possess a simple eigenvalue that crosses the imaginary axis when the Reynolds number passes through a (suitably defined) critical value. We also show that only supercritical or subcritical bifurcation may occur. Our approach is different and, we believe, more direct than those used by previous authors in similar, but distinct, context.
\end{abstract}

\section{Introduction}

One of the most classical phenomena in fluid dynamics is the spontaneous oscillation of the wake in the flow of a viscous liquid past a circular cylinder. More precisely, suppose that a cylinder, $\mathscr{C}$, of diameter $d$ is placed with its axis a orthogonal to the flow of a viscous liquid having an upstream constant velocity $\boldsymbol{v}_{\infty}$. Let $\lambda:=\left|\boldsymbol{v}_{\infty}\right| /(\nu d)$ be the relevant Reynolds number of the flow, with $\nu$ kinematic viscosity of the liquid. It is then experimentally observed that there is a critical value, $\lambda_{0} \sim 50$, such that if $\lambda<\lambda_{0}$ the motion of the liquid in a region sufficiently far from the ends of $\mathscr{C}$ that includes $\mathscr{C}$, is planar, steady and stable, whereas as soon as $\lambda>\lambda_{0}$, the motion is still planar, but its regime becomes oscillatory, as evidenced by the time-periodic

${ }^{*}$ Department of Mechanical Engineering and Materials Science, University of Pittsburgh, PA 15261. Work partially supported by NSF DMS Grant-1311983. 
motion of the wake behind $\mathscr{C}$; 37, Chapter 3]. It is worth emphasizing that the unsteadiness of the flow arises spontaneously even though the imposed condition -uniform flow at far distances- is time-independent.

This is a beautiful and clear example of what, in mathematical terms, is defined as time-periodic bifurcation. The main objective of this paper is to provide a rigorous analysis of this interesting phenomenon.

In this respect, we begin to recall that, from the mathematical viewpoint, this means to investigate the following set of (dimensionless) equations

$$
\begin{gathered}
\partial_{t} \boldsymbol{V}+\lambda\left(\boldsymbol{V}-\boldsymbol{e}_{1}\right) \cdot \nabla \boldsymbol{V}=\Delta \boldsymbol{V}-\nabla P \\
\operatorname{div} \boldsymbol{V}=0 \\
\boldsymbol{V}=\boldsymbol{e}_{1} \text { at } \partial \Omega \times \mathbb{R},
\end{gathered}
$$

with the further condition

$$
\lim _{|x| \rightarrow \infty} \boldsymbol{V}(x, t)=\mathbf{0}, \quad t \in \mathbb{R} .
$$

Here $\boldsymbol{V}$ and $P$ are velocity and pressure fields of the liquid, $\Omega$ is the relevant two-dimensional unbounded region of flow (the entire portion of the plane outside the normal cross-section of $\mathscr{C}$ ), and $e_{1}$ is a unit vector parallel to $\boldsymbol{v}_{\infty}$. It is known that, under suitable assumptions on $\lambda_{0}$, the above equations possess a unique steady-state solution branch $(\boldsymbol{u}(\lambda), p(\lambda))$, with $\lambda$ in a neighborhood $U\left(\lambda_{0}\right)$ [11]. Writing $\boldsymbol{V}=\boldsymbol{v}(x, t ; \lambda)+\boldsymbol{u}(x ; \lambda), P=\mathrm{p}(x, t ; \lambda)+p(x ; \lambda)$, equations (1.1)-(1.2) become

$$
\left.\begin{array}{c}
\partial_{\tau} \boldsymbol{v}+\lambda\left[\left(\boldsymbol{v}-\boldsymbol{e}_{1}\right) \cdot \nabla \boldsymbol{v}+\boldsymbol{u}(\lambda) \cdot \nabla \boldsymbol{v}+\boldsymbol{v} \cdot \nabla \boldsymbol{u}(\lambda)\right]=\Delta \boldsymbol{v}-\nabla \mathrm{p} \\
\operatorname{div} \boldsymbol{v}=0
\end{array}\right\} \text { in } \Omega \times \mathbb{R}
$$

with

$$
\lim _{|x| \rightarrow \infty} \boldsymbol{v}(x, t)=\mathbf{0}, \quad t \in \mathbb{R} .
$$

Our bifurcation problem consists then in finding sufficient conditions for the existence of a non-trivial family of time-periodic solutions to (1.3)-(1.4), $(\boldsymbol{v}(\lambda), \mathrm{p}(\lambda)), \lambda \in U\left(\lambda_{0}\right)$, of period $T=T(\lambda)$ (unknown as well), such that $(\boldsymbol{v}(t ; \lambda), \nabla \mathrm{p}(t ; \lambda)) \rightarrow(\mathbf{0}, \mathbf{0})$ as $\lambda \rightarrow \lambda_{0}$.

In order to better understand the heart of the problem and the motivation behind our approach, we begin to observe that, formally, (1.3)-(1.4) can be thought of as a special case of evolution equations of the type

$$
\frac{d u}{d t}=N(\lambda, u), \quad t \in \mathbb{R}
$$


where $N:(\lambda, u) \in U\left(\lambda_{0}\right) \times X \mapsto Y$ (X,Y Banach spaces $)$ is a smooth nonlinear operator with $N(\lambda, 0)=0$, for all $\lambda \in U\left(\lambda_{0}\right)$. The objective is then to find a family of time-periodic solutions $u=u(t ; \lambda)$ of period $T=T(\lambda), \lambda \in U\left(\lambda_{0}\right)$, such that $u(\lambda) \rightarrow 0$ as $\lambda \rightarrow \lambda_{0}$. Let $\mathscr{L}=\mathscr{L}(\lambda)$ be the linearization of $N(\lambda, \cdot)$ around $u=0$. Following the original ideas of E. Hopf [19], one has in mind to employ the Implicit Function Theorem, so that the bifurcation problem boils down to find conditions on $\lambda_{0}$, and $T\left(\lambda_{0}\right)$ ensuring that the operator

$$
\frac{d}{d t}-\mathscr{L}\left(\lambda_{0}\right)
$$

is continuously invertible in a suitable class of time-periodic functions. The latter implies that, in particular, the operator $\mathscr{L}\left(\lambda_{0}\right)$ must enjoy this property as well.

The first comprehensive investigation of time-periodic bifurcation may be traced back to the influential work of E. Hopf [19] in the case $X=Y=\mathbb{R}^{n}$ (1) There, Hopf shows the occurrence of bifurcation under a set of conditions that can be roughly summarized as follows: (C1) 0 is not an eigenvalue of $L_{0}:=\mathscr{L}\left(\lambda_{0}\right)$, which ensures, in particular, that $L_{0}$ is continuously invertible; (C2) $L_{0}$ possesses two and only two purely imaginary eigenvalues $\pm \mathrm{i} \omega_{0}(\neq 0)$ that are also simple, and (C3) As $\lambda$ passes through $\lambda_{0}$, the eigenvalues of $\mathscr{L}(\lambda)$ "cross" the imaginary axis with nonzero speed.

The approach introduced by Hopf, lends itself to a natural extension to the infinite-dimensional case, at least when the underlying function space has a Hilbert structure, and $L_{0}$ is the generator of an analytic semigroup, with compact resolvent.

Along these lines of thought, Iudovich [22], Joseph \& Sattinger [23], and Iooss [20] pioneered the investigation of the occurrence of self-oscillation in a viscous flow in a bounded domain. More precisely, they furnished sufficient conditions, basically of the same type as those listed above, for the existence (and uniqueness) of bifurcating time-periodic solutions from steady-state solutions to the Navier-Stokes equations. (2)

It is important to emphasize that the assumption that the flow occurs in a bounded domain is fundamental. In fact, it ensures, among other things, that $L_{0}$ has a purely discrete spectrum which, in turn, implies that if 0 is not an eigenvalue then $L_{0}$ has a bounded inverse.

\footnotetext{
${ }^{(1)}$ However, see also the previous contributions of Poincaré [35] and Andronov \& Witt [1.

${ }^{(2)}$ For further development of the theory, its generalization and major applications to the Navier-Stokes equations, we refer to, e.g., [7, 21, 6, 40, 26] and the reference therein.
} 
In the case of an exterior domain (flow past an obstacle), $L_{0}$ assumes the following form

$$
L_{0}(\boldsymbol{v}):=\mathrm{P}\left[\Delta \boldsymbol{v}+\lambda_{0}\left(\boldsymbol{e}_{1} \cdot \nabla \boldsymbol{v}-\boldsymbol{u}\left(\lambda_{0}\right) \cdot \nabla \boldsymbol{v}-\boldsymbol{v} \cdot \nabla \boldsymbol{u}\left(\lambda_{0}\right)\right)\right],
$$

where $\mathrm{P}$ is the Helmholtz projection (3) However, when defined in its "natural space", namely, the subspace, $Z^{2, q}, 1<q<\infty$, of solenoidal functions from the Sobolev space $W^{2, q}$ with zero trace at the boundary, the operator $L_{0}$ in (1.6) shows a non-empty essential spectrum, and worse, 0 is a point of this spectrum for all $\lambda_{0}$ [4, 8]. As a result, the property of continuous invertibility of $L_{0}$ is no longer secured.

Nonetheless, if we define $L_{0}$ on an appropriate homogeneous Sobolev space, $\mathcal{B}$, then we can show that $L_{0}$ is Fredholm of index 0 (see [15, Theorem 3.1], [11, Theorem 1.8]), so that, in this framework, bounded invertibility is again guaranteed by requiring that 0 is not an eigenvalue. These observations suggest that for flow past an obstacle, the study of (1.3)-(1.4) and the associated time-periodic bifurcation problem should be performed in the Banach space, $\mathcal{B}$, where $L_{0}$ enjoys the Fredholm property. This is, in fact, the approach employed by Babenko [5], successively revisited and extended in a non-trivial way by Sazonov [36], also along the ideas of the seminal paper 22] We wish to emphasize that the methods used by these authors work in dimension $n=3$, whereas they do not admit any sensible generalization to the case $n=2$; see, e.g., [3, p.39].

It is worth remarking that the space $\mathcal{B}$ above is a subclass of the space where steady-state solutions to (1.1)-(1.2) exist. In this respect, we recall that it is a standard procedure, for flow in exterior domains, to formulate time-periodic problems in function spaces where steady-state solutions exist; see, e.g., [31, 32, 28, 38, 16, 24, 34] and the reference therein. However, as first pointed out in [13, 14] even though "natural" at first sight ("steady state solution is a special case of a time-periodic one"), this formulation is not convenient, and, in fact, as detailed in [14, is unable to cover the two-dimensional problem of flow past a cylinder, which is the focus of this paper. In view of these considerations, in [13, 14] we introduced a different method that, essentially, consists in reformulating the original problem as a coupled nonlinear system constituted by an elliptic equation, formulated in the "natural" space of steady-state solutions, and a parabolic equation that can be framed in a much "better" space; see also the analysis of Kyed in 27.

\footnotetext{
${ }^{(3)}$ For notation, see the next section.

${ }^{(4)}$ See also Melcher et al. 33 for a whole-space, vorticity formulation.
} 
The approach we propose to study time-periodic bifurcation stems from the one introduced in [14] and, in our opinion, is simpler and more direct than those of [5, 36], with the further advantage that it allows us to cover the two-dimensional case. Basically, it consists of two main steps. We first split the sought solution, $u$, as sum of its time average over a period, $u^{(1)}$, and of its "purely periodic" component, $u^{(2)}$, with zero average. Accordingly, the original problem (1.5) then splits into a coupled (nonlinear) "ellipticparabolic" system in the unknowns $u^{(1)}$ and $u^{(2)}$, of the form

$$
L_{0}\left(u^{(1)}\right)=N_{1}\left(\lambda, u^{(1)}, u^{(2)}\right), \quad \frac{d}{d t} u^{(2)}-L_{0}\left(u^{(2)}\right)=N_{2}\left(\lambda, u^{(1)}, u^{(2)}\right),
$$

where $N_{i}, i=1,2$, are suitable (smooth) nonlinear operators. Now, the crucial point that makes our method different than those of [5, 36], is that, in spite of the fact that the operator $L_{0}$ is formally the same (see (1.6)) we frame the two equations in (1.7) in two different function spaces, by choosing domains D and ranges $\mathrm{R}$ of $L_{0}$ appropriately. Specifically, in (1.7) 1 we take $L_{0} \equiv \widetilde{\mathscr{L}_{0}}$ with $\mathrm{D}$ coinciding with the "natural" Banach space $\mathcal{B}$ of steady-state solutions and $\mathrm{R} \subseteq L^{q}$, for suitable $q>1$, whereas in (1.7) 2 , we pick $L_{0} \equiv \mathscr{L}_{0}$, with $\mathrm{D}:=Z^{2,2}$, and $\mathrm{R} \subseteq L^{2}$. Once the steady-state part $u^{(1)}$ of the solution has been "isolated" in the sense specified above, we can then show that the bifurcation problems reduces, essentially, to the study of the property of the parabolic operator $d u / d t-\mathscr{L}_{0}(u)$ in $(1.5)_{2}$ in the standard $L^{2}$ context, exactly as in the case of a bounded region of flow [22, 23]. For this reason, it presents no further conceptual difficulties.

Although our approach could be applied to a vast class of evolutionary equations, we shall employ it here to study time-periodic bifurcation from a steady-state Navier-Stokes flow past a cylinder. More specifically, under the assumptions (ㅍ1) $-(\mathrm{H} 3)$ formulated later on in the paper -which resemble conditions $(\mathrm{C} 1)-(\mathrm{C} 3)$ of the original paper of Hopf- we show, by means of the implicit function theorem, the existence of a one-parameter family of timeperiodic solutions, branching out the steady-state solution $\mathrm{s}_{0}$ at $\lambda=\lambda_{0}$; see Theorem 4.1(a). A characteristic feature of these solutions is that they exist either for $\lambda<\lambda_{0}$ or for $\lambda>\lambda_{0}$, so that the bifurcation is either subcritical or supercritical; see Theorem 4.1(c). Moreover, we prove that (up to a phase shift) any other time-periodic solution branching out of $\left(\lambda_{0}, \mathrm{~s}_{0}\right)$ must belong to the above family, under a further assumption on the branching frequency that is validated by numerical tests; see Theorem 4.1(b) and Theorem 5.1 .

In more detail, the plan of the paper is the following. After introducing some basic notation in Section 2, in the following Section 3 our main objective is to analyze the relevant properties of the linearized operators $\widetilde{\mathscr{L}}_{0}$ and 
$\mathscr{L}_{0}$. To this end, we recall in Proposition 3.1 that $\widetilde{\mathscr{L}_{0}}$, when defined in the classical homogeneous and anisotropic Sobolev space of steady-state solutions to (1.4)-(1.4), is Fredholm of index 0. This circumstance is supportive of our first assumption (H1), namely, that the null space of $\widetilde{\mathscr{L}_{0}}$ is trivial, so that $\widetilde{\mathscr{L}_{0}}$ is boundedly invertible. Successively, we analyze the properties of the operator $\mathscr{L}_{0}$ in a subspace of the Sobolev space $W^{2,2}$, and its "parabolic" counterpart, $\mathscr{Q}(u):=d u / d t-\mathscr{L}_{0}(u)$, in the space $\mathscr{W}_{2 \pi, 0}^{2}$ of maximal $L^{2}$-regularity of $2 \pi$-periodic functions with zero average over a period. In this respect, in Proposition 3.3, we prove that $\mathscr{L}_{0}$ may have an at most countable number of purely imaginary eigenvalues that can only cluster at 0 ; moreover, each of these eigenvalues is isolated and of finite algebraic multiplicity. This provides the basis, on the one hand, of our assumption (H2) that requires, in particular, that $\mathscr{L}_{0}$ has a simple, purely imaginary eigenvalue. On the other hand, by resorting to a classical result on perturbations of simple eigenvalues (see Proposition 3.5), it also supports assumption ( $\mathrm{H3}$ ) regarding the way in which the eigenvalues of $\mathscr{L}(\lambda)$ "cross" the imaginary axis when $\lambda$ passes $\lambda_{0}$. We then study the properties of the operator $\mathscr{Q}$, and show that it is Fredholm of index 0 (Lemma 3.5). The latter, combined with assumption ( $\mathrm{H} 2$ ) allows us to give necessary and sufficient conditions for the bounded invertibility of $\mathscr{Q}$. With these results in hand, in Section 4, Theorem 4.1, under the assumptions ( $\mathrm{H1}$ - $(\mathrm{H3})$ we prove the result of existence of a one-parameter family of time-periodic solutions to (1.3)-(1.4) mentioned earlier on, with the property of being either subcritical or supercritical. Finally, the uniqueness property of these solutions is discussed, in full generality, in Section 5; see Theorem 5.1.

\section{Notation}

We let $\mathbb{N}, \mathbb{Z}$, and $\mathbb{R}, \mathbb{C}$ represent, in the order, the sets of positive and relative integers, and the fields of real and complex numbers. Thus, $\mathbb{R}^{2}$ indicates the whole plane. The canonical base in $\mathbb{R}^{2}$ is denoted by $\mathfrak{B}:=\left\{\boldsymbol{e}_{1}, \boldsymbol{e}_{2}\right\}$. A vector $\boldsymbol{u}$ will have two components in $\mathfrak{B}$, denoted by $u_{1}$ and $u_{2}$, respectively. Likewise, coordinates of a point $x \in \mathbb{R}^{2}$ in the frame $\left\{O, \boldsymbol{e}_{1}, \boldsymbol{e}_{2}\right\}, O \in \mathbb{R}^{2}$, will be indicated by $x_{1}, x_{2}$.

$\Omega$ stands for a fixed planar exterior domain, namely, the complement of the closure of a bounded, open, and simply connected set, $\Omega_{0}$, of $\mathbb{R}^{2}$. We shall assume $\Omega$ of class $C^{2}$. Moreover, we take the origin $O$ of the coordinate system in $\Omega_{0}$, and denote by $R_{*}>0$ a number such that the closure of $\Omega_{0}$ is strictly contained in the disk $\left\{\boldsymbol{x} \in \mathbb{R}^{2}:\left(x_{1}^{2}+x_{2}^{2}\right)^{\frac{1}{2}}<R_{*}\right\}$. 
For $R \geq R_{*}$, we let

$$
\Omega_{R}=\Omega \cap\left\{\boldsymbol{x} \in \mathbb{R}^{2}:\left(x_{1}^{2}+x_{2}^{2}\right)^{\frac{1}{2}}<R\right\}, \quad \Omega^{R}=\Omega-\overline{\Omega_{R}},
$$

where the bar denotes closure.

We set $\partial_{t} \boldsymbol{u}:=\partial \boldsymbol{u} / \partial t, \partial_{1} \boldsymbol{u}:=\partial \boldsymbol{u} / \partial x_{1}$, and indicate by $D^{2} \boldsymbol{u}$ the matrix of the second derivatives of $\boldsymbol{u}$.

For an open and connected set $A \subseteq \mathbb{R}^{2}, L^{q}(A), L_{l o c}^{q}(A), 1 \leq q \leq \infty$, $W^{m, q}(A), W_{0}^{m, q}(A), m \geq 0,\left(W^{0, q} \equiv W_{0}^{0, q} \equiv L^{q}\right)$, stand for the usual Lebesgue and Sobolev classes, respectively, of real or complex functions. (5) Norms in $L^{q}(A)$ and $W^{m, q}(A)$ are indicated by $\|\cdot\|_{q, A}$ and $\|\cdot\|_{m, q, A}$. The scalar product of functions $u, v \in L^{2}(A)$ will be denoted by $\langle u, v\rangle_{A}$. In the above notation, the symbol $A$ will be omitted, unless confusion arises.

As customary, for $q \in[1, \infty]$ we let $q^{\prime}=q /(q-1)$ be its Hölder conjugate.

By $D^{1, q}(\Omega), 1<q<\infty$, we denote the space of (equivalence classes of) functions $u$ such that $\|\nabla u\|_{q}<\infty$. Moreover, setting,

$$
\mathcal{D}(\Omega):=\left\{\boldsymbol{u} \in C_{0}^{\infty}(\Omega): \operatorname{div} \boldsymbol{u}=0\right\}
$$

we let $\mathcal{D}_{0}^{1,2}(\Omega)$ be the completion of $\mathcal{D}(\Omega)$ in the norm $\|\nabla(\cdot)\|_{2}$, and set

$$
Z^{2,2}(\Omega):=W^{2,2}(\Omega) \cap \mathcal{D}_{0}^{1,2}(\Omega) .
$$

Furthermore, we denote by $H_{q}(\Omega), 1<q<\infty,\left(H_{2}(\Omega) \equiv H(\Omega)\right)$ the completion of $\mathcal{D}(\Omega)$ in the norm $L^{q}(\Omega)$ and let $\mathrm{P}_{q}$ be the (Helmholtz) projection from $L^{q}(\Omega)$ onto $H_{q}(\Omega)$. $\mathrm{P}_{q}$ is independent of $q[12$, $\S I I I .1]$, so that we shall simply denote it by $\mathrm{P}$.

For $q \in(1,3 / 2)$, we define

$$
\begin{gathered}
X^{2, q}(\Omega):=\left\{\boldsymbol{u} \in L_{l o c}^{1}\left(\mathbb{R}^{2}\right): u_{2} \in L^{\frac{2 q}{2-q}}(\Omega), \nabla u_{2}, \partial_{1} \boldsymbol{u}, D^{2} \boldsymbol{u} \in L^{q}(\Omega),\right. \\
\left.\boldsymbol{u} \in L^{\frac{3 q}{3-2 q}}(\Omega), \nabla \boldsymbol{u} \in L^{\frac{3 q}{3-q}}(\Omega)\right\} .
\end{gathered}
$$

and

$$
X_{0}^{2, q}(\Omega):=\left\{\boldsymbol{u} \in X^{2, q}(\Omega): \operatorname{div} \boldsymbol{u}=0,\left.\boldsymbol{u}\right|_{\partial \Omega}=\mathbf{0}\right\} .
$$

As is known, $X^{2, q}(\Omega)$ and $X_{0}^{2, q}(\Omega)$ become Banach spaces when endowed with the "natural" norm

$$
\|\boldsymbol{u}\|_{X^{2, q}}:=\left\|u_{2}\right\|_{\frac{2 q}{2-q}}+\left\|\nabla u_{2}\right\|_{q}+\left\|u_{1}\right\|_{\frac{3 q}{3-2 q}}+\|\nabla \boldsymbol{u}\|_{\frac{3 q}{3-q}}+\left\|\partial_{1} \boldsymbol{u}\right\|_{q}+\left\|D^{2} \boldsymbol{u}\right\|_{q}
$$

\footnotetext{
${ }^{(5)}$ We shall use the same font style to denote scalar, vector and tensor function spaces.
} 
see [12, §XII.5].

If $M$ is a map between two spaces, we denote by $\mathrm{N}[M]$ and $\mathrm{R}[M]$ its null space and range, respectively.

In the following, $B$ is a real Banach space with associated norm $\|\cdot\|_{B}$.

By $B_{\mathbb{C}}:=B+\mathrm{i} B$ we denote the complexification of $B$.

For $q \in[1, \infty], L^{q}(0,2 \pi ; B)$ is the space of functions $u:(0,2 \pi) \rightarrow B$ such that

$$
\left(\int_{0}^{2 \pi}\|u(t)\|_{B}^{q}\right)^{\frac{1}{q}}<\infty, \quad \text { if } q \in[1, \infty) ; \quad \underset{t \in[0,2 \pi]}{\operatorname{ess} \sup }\|u(t)\|_{B}<\infty, \quad \text { if } q=\infty .
$$

Also, by $C(0,2 \pi ; B)$ we indicate the set of functions $u:[0,2 \pi] \rightarrow B$ which are continuous from $[0,2 \pi]$ with values in $B$.

Given a function $u:(x, t) \in \Omega \times[0,2 \pi] \rightarrow \mathbb{R}^{2}$, we let $\bar{u}=\bar{u}(x)$ be its average over $[0,2 \pi]$, namely,

$$
\bar{u}(x):=\frac{1}{2 \pi} \int_{0}^{2 \pi} u(x, t) d t .
$$

Furthermore, we shall say that $u$ is $2 \pi$-periodic, if $u(x, 0)=u(x, 2 \pi)$, for a.a. $x \in \Omega$. Clearly, such functions can be extended periodically to all $t \in \mathbb{R}$. We then define

$$
\begin{aligned}
\mathscr{W}_{2 \pi, 0}^{2}(\Omega):=\left\{\boldsymbol{u} \in W^{1,2}(0,2 \pi ; H(\Omega))\right. & \cap L^{2}\left(0,2 \pi ; Z^{2,2}(\Omega)\right): \\
& \boldsymbol{u} \text { is } 2 \pi \text {-periodic with } \overline{\boldsymbol{u}}=\mathbf{0}\}
\end{aligned}
$$

with associated norm

$$
\|u\|_{\mathscr{W}_{2 \pi, 0}^{2}}:=\left(\int_{0}^{2 \pi}\left\|\partial_{t} \boldsymbol{u}(t)\right\|_{2}^{2} d t\right)^{1 / 2}+\left(\int_{0}^{2 \pi}\|\boldsymbol{u}(t)\|_{2,2}^{2} d t\right)^{1 / 2} .
$$

Likewise, setting

$$
\Omega_{2 \pi}:=\Omega \times[0,2 \pi]
$$

we define

$$
\left.\mathscr{L}_{2 \pi, 0}(\Omega):=\left\{\boldsymbol{u} \in L^{2}\left(\Omega_{2 \pi}\right)\right): \boldsymbol{u} \text { is } 2 \pi \text {-periodic with } \overline{\boldsymbol{u}}=\mathbf{0}\right\},
$$

and its subspace

$$
\mathscr{H}_{2 \pi, 0}(\Omega):=\left\{\boldsymbol{u} \in L^{2}(0,2 \pi ; H(\Omega)): \boldsymbol{u} \text { is } 2 \pi \text {-periodic with } \overline{\boldsymbol{u}}=\mathbf{0}\right\} .
$$


Moreover, for $\boldsymbol{u}, \boldsymbol{v} \in \mathscr{L}_{2 \pi, 0}^{2}(\Omega)$ we put

$$
(\boldsymbol{u} \mid \boldsymbol{v}):=\int_{0}^{2 \pi}\langle\boldsymbol{u}(t), \boldsymbol{v}(t)\rangle d t
$$

Finally, by $c, c_{0}, c_{1}$, etc., we denote positive constants, whose particular value is unessential to the context. When we wish to emphasize the dependence of $c$ on some parameter $\xi$, we shall write $c(\xi)$.

\section{Analysis of the Relevant Linearized Operators}

Objective of this section is to introduce some relevant linear operators and study their main properties in different function spaces.

To this end, for $\lambda_{0}>0$ and a given (sufficiently smooth) vector field $\boldsymbol{u}_{0}=\boldsymbol{u}_{0}(x)$, we consider the following operator, which can be viewed as a "perturbation" to the classical Oseen operator:

$$
\boldsymbol{v} \mapsto \mathrm{P}\left[\Delta \boldsymbol{v}+\lambda_{0}\left(\partial_{1} \boldsymbol{v}-\boldsymbol{u}_{0} \cdot \nabla \boldsymbol{v}+\boldsymbol{v} \cdot \nabla \boldsymbol{u}_{0}\right)\right]
$$

The next result is shown in [11, Theorem 1.8].

Proposition 3.1 Let $\boldsymbol{u}_{0} \in X^{2, q}(\Omega), q \in(1,6 / 5]$. Then

$$
\widetilde{\mathscr{L}_{0}}: \boldsymbol{v} \in X_{0}^{2, q}(\Omega) \mapsto P\left[\Delta \boldsymbol{v}+\lambda_{0}\left(\partial_{1} \boldsymbol{v}-\boldsymbol{u}_{0} \cdot \nabla \boldsymbol{v}+\boldsymbol{v} \cdot \nabla \boldsymbol{u}_{0}\right)\right] \in H_{q}(\Omega)
$$

is Fredholm of index 0 .

With the help of this proposition one can show the following one, whose proof can be found in [11, Theorem 2.3].

Proposition 3.2 Assume that $\left(\boldsymbol{u}_{0}, p_{0}\right) \in X^{2, q}(\Omega) \times D^{1, q}(\Omega), 1<q<6 / 5$, is a solution to the steady-state problem

$$
\begin{gathered}
\left.\begin{array}{c}
\Delta \boldsymbol{u}+\lambda \partial_{1} \boldsymbol{u}=\lambda \boldsymbol{u} \cdot \nabla \boldsymbol{u}+\nabla p \\
\operatorname{div} \boldsymbol{u}=0
\end{array}\right\} \text { in } \Omega \\
\boldsymbol{u}=\boldsymbol{e}_{1} \text { at } \partial \Omega, \quad \lim _{|x| \rightarrow \infty} \boldsymbol{u}(x)=\mathbf{0},
\end{gathered}
$$

with $\lambda=\lambda_{0}$. Then, if

$$
\mathrm{N}\left[\widetilde{\mathscr{L}_{0}}\right]=\{0\},
$$


problem (3.3) has a solution that is (real) analytic at $\lambda=\lambda_{0}$. Precisely, there is a neighborhood $U\left(\lambda_{0}\right)$ of $\lambda_{0}$ and a family of solutions to (3.3), $(\boldsymbol{u}(\lambda), p(\lambda)) \in X^{2, q}(\Omega) \times D^{1, q}(\Omega), \lambda \in U\left(\lambda_{0}\right)$, such that the series

$$
\boldsymbol{u}(\lambda)=\boldsymbol{u}_{0}+\sum_{k=1}^{\infty}\left(\lambda-\lambda_{0}\right)^{k} \boldsymbol{u}_{k}, \quad p(\lambda)=p_{0}+\sum_{k=1}^{\infty}\left(\lambda-\lambda_{0}\right)^{k} p_{k}
$$

are absolutely convergent in $X^{2, q}(\Omega)$ and $D^{1, q}(\Omega)$, respectively.

We now consider the operator (3.1) with domain of definition $Z^{2,2}(\Omega) \subset$ $H(\Omega)$ and values in $H(\Omega)$, and denote it by $\mathscr{L}_{0}$. Since $Z^{2,2}(\Omega)$ is dense in $H(\Omega), \mathscr{L}_{0}$ is densely defined. We are interested to establish certain important properties of the spectrum $\sigma\left(\mathscr{L}_{0}\right)$. To do this, we extend $\mathscr{L}_{0}$ to a linear operator (still denoted by $\mathscr{L}_{0}$ ) on $Z_{\mathbb{C}}^{2,2}(\Omega)$ and $H_{\mathbb{C}}(\Omega)$ :

$$
\mathscr{L}_{0}: \mathrm{D}_{\mathbb{C}}\left(\mathscr{L}_{0}\right) \subset H_{\mathbb{C}}(\Omega) \mapsto H_{\mathbb{C}}(\Omega), \quad \mathrm{D}_{\mathbb{C}}\left(\mathscr{L}_{0}\right):=Z_{\mathbb{C}}^{2,2}(\Omega) .
$$

We shall then show, in particular, that the intersection of $\sigma\left(\mathscr{L}_{0}\right)$, with $\{\mathrm{i} \mathbb{R}-$ $\{0\}\}$ can only be constituted by a finite or countable number of eigenvalues with finite multiplicity (see Proposition 3.3).

The proof of this property requires a number of preparatory results.

Lemma 3.1 Let $\omega \in \mathbb{R}-\{0\}$. Then, for a given $f \in L_{\mathbb{C}}^{2}(\Omega)$ there is a unique corresponding $(\boldsymbol{u}, p) \in W_{\mathbb{C}}^{2,2}(\Omega) \times D_{\mathbb{C}}^{1,2}(\Omega)$ such that

$$
\left.\begin{array}{c}
\Delta \boldsymbol{u}+\lambda_{0} \partial_{1} \boldsymbol{u}-\mathrm{i} \omega \boldsymbol{u}=\boldsymbol{f}+\nabla p \\
\operatorname{div} \boldsymbol{u}=0 \\
\boldsymbol{u}=\mathbf{0} \text { at } \partial \Omega .
\end{array}\right\} \text { in } \Omega,
$$

Moreover, there are constants $c$ and $c_{0}$ depending only on $\Omega$, such that $(\boldsymbol{u}, p)$ satisfies the following inequality

$$
\left\|D^{2} \boldsymbol{u}\right\|_{2}+|\omega|^{\frac{1}{2}}\|\nabla \boldsymbol{u}\|_{2}+|\omega|\|\boldsymbol{u}\|_{2}+\|\nabla p\|_{2} \leq c\|\boldsymbol{f}\|_{2}, \quad|\omega| \geq \max \left\{\lambda_{0}^{2}, 1\right\} .
$$

Proof. If we dot-multiply both sides of $(3.6)_{1}$ by $\boldsymbol{u}^{*}$ (* $:=$ complex conjuga- $^{*}$ tion), integrate by parts over $\Omega$ and use $(3.6)_{2,3}$, we formally obtain

$$
-\|\nabla \boldsymbol{u}\|_{2}^{2}-\mathrm{i} \omega\|\boldsymbol{u}\|_{2}^{2}=\left\langle\boldsymbol{f}, \boldsymbol{u}^{*}\right\rangle-\lambda_{0}\left\langle\partial_{1} \boldsymbol{u}, \boldsymbol{u}^{*}\right\rangle .
$$

By separating real and imaginary parts, and applying Schwartz inequality we infer

$$
\begin{aligned}
\|\nabla \boldsymbol{u}\|_{2}^{2} & \leq\|\boldsymbol{u}\|_{2}\|\boldsymbol{f}\|_{2} \\
|\omega|\|\boldsymbol{u}\|_{2} & \leq \lambda_{0}\|\nabla \boldsymbol{u}\|_{2}+\|\boldsymbol{f}\|_{2}
\end{aligned}
$$


Replacing (3.8) ${ }_{1}$ into (3.8) 2 and using Cauchy-Schwartz inequality, we easily show that

$$
|\omega|\|\boldsymbol{u}\|_{2} \leq\|\boldsymbol{f}\|_{2}+\lambda_{0}\|\boldsymbol{u}\|_{2}^{\frac{1}{2}}\|\boldsymbol{f}\|_{2}^{\frac{1}{2}} \leq\left(1+\frac{\lambda_{0}^{2}}{2|\omega|}\right)\|\boldsymbol{f}\|_{2}+\frac{|\omega|}{2}\|\boldsymbol{u}\|_{2}
$$

which implies

$$
|\omega|\|\boldsymbol{u}\|_{2} \leq\left(2+\frac{\lambda_{0}^{2}}{|\omega|}\right)\|\boldsymbol{f}\|_{2} .
$$

Replacing this time (3.9) into (3.8) 1 we also show

$$
|\omega|^{\frac{1}{2}}\|\nabla \boldsymbol{u}\|_{2} \leq\left(2+\frac{\lambda_{0}^{2}}{|\omega|}\right)^{\frac{1}{2}}\|\boldsymbol{f}\|_{2} .
$$

By means of the latter two estimates in conjunction with the classical Galerkin method, one can prove by standard arguments the existence of a (weak) solution to (3.6) $)(\boldsymbol{u}, p) \in W_{\mathbb{C}}^{1,2}(\Omega) \times L_{\text {loc }}^{2}\left(\Omega_{R}\right)$ for all $R>R_{*}$; see, e.g. [12, §VII.2]. We now write (3.6) as the following Stokes problem

$$
\left.\begin{array}{rl}
\Delta \boldsymbol{u} & =\boldsymbol{F}+\nabla p \\
\operatorname{div} \boldsymbol{u} & =0 \\
\boldsymbol{u} & =\mathbf{0} \text { at } \partial \Omega .
\end{array}\right\} \text { in } \Omega,
$$

with $\boldsymbol{F}:=\lambda_{0} \partial_{1} \boldsymbol{u}-\mathrm{i} \omega \boldsymbol{u}+\boldsymbol{f}$. In view of (3.9)-(3.10) we get $\boldsymbol{F} \in L_{\mathbb{C}}^{2}$, so that, by well-known results [12, Theorem IV.5.1, Theorem V.5.3(ii)] we deduce that $(\boldsymbol{u}, p) \in Z_{\mathbb{C}}^{2,2}(\Omega) \times D_{\mathbb{C}}^{1,2}(\Omega)$. The existence property is thus secured. As for uniqueness in the class $Z_{\mathbb{C}}^{2,2}$, it is readily established. In fact, it is enough to proceed as in the proof of (3.9) $-(3.10)$ with $\boldsymbol{f} \equiv \mathbf{0}$. It remains to show the validity of (3.7). To this end, we observe that, since, obviously

$$
\|\boldsymbol{F}\|_{2} \leq\left(\lambda_{0}\|\nabla \boldsymbol{u}\|_{2}+|\omega|\|\boldsymbol{u}\|_{2}+\|\boldsymbol{f}\|_{2}\right),
$$

from [12, Remark IV.4.2, Lemma V.4.3] we deduce

$$
\|\nabla p\|_{2}+\left\|D^{2} \boldsymbol{u}\right\|_{2} \leq c\left(\lambda_{0}\|\nabla \boldsymbol{u}\|_{2}+|\omega|\|\boldsymbol{u}\|_{2}+\|\boldsymbol{f}\|_{2}+\|\boldsymbol{u}\|_{2, \Omega_{R_{*}}}\right),
$$

with some $c=c(\Omega)$. Inequality (3.7) then follows, under the stated assumptions on $|\omega|$, from the latter and (3.9)-(3.10). The lemma is completely proved. 
Lemma 3.2 The operator

$$
\mathscr{K}: \boldsymbol{v} \in Z^{2,2}(\Omega) \mapsto \boldsymbol{u}_{0} \cdot \nabla \boldsymbol{v}+\boldsymbol{v} \cdot \nabla \boldsymbol{u}_{0} \in L^{2}(\Omega)
$$

is compact.

Proof. We begin to observe that the embeddings

$$
\left.\begin{array}{l}
Z^{2,2}(\Omega) \subset W^{1,2}\left(\Omega_{R}\right) \\
W^{1,2}\left(\Omega_{R}\right) \subset L^{r}(\Omega), \quad r \in(1, \infty)
\end{array}\right\} \text { are compact, for all } R>R_{*} .
$$

Now, let $\left\{\boldsymbol{v}_{n}\right\} \subset Z^{2,2}(\Omega)$ with $\left\|\boldsymbol{v}_{n}\right\|_{2,2}=1$, for all $n \in \mathbb{N}$, and let $\overline{\boldsymbol{v}} \in Z^{2,2}(\Omega)$ be its weak limit. Without loss of generality, we may assume $\overline{\boldsymbol{v}}=\mathbf{0}$, which gives $\mathscr{K}(\overline{\boldsymbol{v}})=\mathbf{0}$. For any $R>R_{*}$ we show that

$$
\left\|\boldsymbol{u}_{0} \cdot \nabla \boldsymbol{v}_{n}\right\|_{2} \leq\left\|\boldsymbol{u}_{0}\right\|_{\infty}\left\|\nabla \boldsymbol{v}_{n}\right\|_{2, \Omega_{R}}+\left\|\boldsymbol{u}_{0}\right\|_{\infty, \Omega^{R}}\left\|\boldsymbol{v}_{n}\right\|_{2,2}
$$

Likewise, by Hölder inequality,

$$
\left\|\boldsymbol{v}_{n} \cdot \nabla \boldsymbol{u}_{0}\right\|_{2} \leq\left\|\nabla \boldsymbol{u}_{0}\right\|_{\frac{2 q}{2-q}}\left\|\boldsymbol{v}_{n}\right\|_{q^{\prime}, \Omega_{R}}+c_{1}\left\|\nabla \boldsymbol{u}_{0}\right\|_{\frac{2 q}{2-q}, \Omega^{R}}\left\|\boldsymbol{v}_{n}\right\|_{2,2} .
$$

Since, by assumption, $\boldsymbol{u}_{0} \in X^{2, q}(\Omega), 1<q<6 / 5$, it follows that $\boldsymbol{u} \in$ $D^{1,2 q /(2-q)}(\Omega)$, on the one hand, and, on the other hand, $\boldsymbol{u}_{0} \in L^{\infty}(\Omega)$ with $\boldsymbol{u}_{0}(x) \rightarrow \mathbf{0}$ uniformly, as $|x| \rightarrow \infty$; see [14, Lemma 1]. As a result by (3.12) -(3.14), and taking $R$ arbitrarily large, we may conclude

$$
\lim _{n \rightarrow \infty}\left\|\mathscr{K}\left(\boldsymbol{v}_{n}\right)\right\|_{2}=0 .
$$

which proves the claimed compactness property of $\mathscr{K}$, and completes the proof of the proposition.

Lemma 3.3 Let $\boldsymbol{u}_{0} \in X^{2, q}(\Omega), 1<q<6 / 5$, and let $\omega \in \mathbb{R}-\{0\}$. Then the operator

$$
\mathscr{L}_{0}-\mathrm{i} \omega I
$$

with $\mathscr{L}_{0}$ defined in (3.5), is Fredholm of index 0 .

Proof. We begin to notice that, as immediately checked, $\mathscr{L}_{0}$ is (graph) closed. In fact, this follows from [25, Theorem 1.11 in Chapter IV], since $\mathscr{L}_{0}=\mathscr{L}_{1}+\mathscr{K}$, where $\mathscr{L}_{1}: Z_{\mathbb{C}}^{2,2}(\Omega) \subset H_{\mathbb{C}}(\Omega) \mapsto H_{\mathbb{C}}(\Omega)$ is obviously closed

\footnotetext{
${ }^{(6)}$ By $I$ we mean the identity operator in $H_{\mathbb{C}}$.
} 
(Lemma 3.1) and, by Lemma 3.2, $\mathscr{K}$ is $\mathscr{L}_{1}$-compact. These two combined properties also show that (3.15) is Fredholm of index 0 (e.g. [18, Theorem XVII.4.3]). The lemma is thus proved.

We are now in a position to show the first main result of this section.

Proposition 3.3 Let $\boldsymbol{u}_{0} \in X^{2, q}(\Omega), 1<q<6 / 5$, and $\mathscr{L}_{0}$ be defined in (3.5). Then $\sigma\left(\mathscr{L}_{0}\right) \cap\{\mathrm{i} \mathbb{R}-\{0\}\}$ consists, at most, of a finite or countable number of eigenvalues, each of which is isolated and of finite (algebraic) multiplicity, that can only accumulate at 0 .

Proof. Set $\mathscr{L}_{\omega}:=\mathscr{L}_{0}-\mathrm{i} \omega I$. By Lemma 3.3 we know that $\mathscr{L}_{\omega}: H_{\mathbb{C}}(\Omega) \mapsto$ $H_{\mathbb{C}}(\Omega)$ is an (unbounded) Fredholm operator of index 0 , for all $\omega \in \mathbb{R}-\{0\}$. Thus, in view of well-known results (e.g. [18, Theorem XVII.2.1]), in order to prove the stated property it is enough to show that there is $\bar{\omega}>0$ such that for all $|\omega|>\bar{\omega}, \mathrm{N}\left[\mathscr{L}_{\omega}\right]=\{0\}$. Now, the equation $\mathscr{L}_{\omega}(\boldsymbol{v})=\mathbf{0}$ is equivalent to the following problem

$$
\left.\begin{array}{rl}
\Delta \boldsymbol{v}+\lambda_{0} \partial_{1} \boldsymbol{v}-\mathrm{i} \omega \boldsymbol{v} & =\lambda_{0}\left(\boldsymbol{u}_{0} \cdot \nabla \boldsymbol{v}+\boldsymbol{v} \cdot \nabla \boldsymbol{u}_{0}\right)+\nabla p \\
\operatorname{div} \boldsymbol{v}= & 0 \\
& \boldsymbol{v}=\mathbf{0} \text { at } \partial \Omega,
\end{array}\right\} \text { in } \Omega,
$$

with $(\boldsymbol{v}, p) \in Z_{\mathbb{C}}^{2,2}(\Omega) \times D_{\mathbb{C}}^{1,2}(\Omega)$. Using Lemma 3.1 and (3.7) in problem (3.16), with the help of Hölder inequality we get, in particular, for all $|\omega| \geq$ $\max \left\{\lambda_{0}^{2}, 1\right\}$,

$$
\begin{aligned}
\left\|D^{2} \boldsymbol{v}\right\|_{2}+|\omega|^{\frac{1}{2}}\|\nabla \boldsymbol{v}\|_{2}+|\omega|\|\boldsymbol{v}\|_{2} & \leq c \lambda_{0}\left\|\boldsymbol{u}_{0} \cdot \nabla \boldsymbol{v}+\boldsymbol{v} \cdot \nabla \boldsymbol{u}_{0}\right\|_{2} \\
& \leq c \lambda_{0}\left(\left\|\boldsymbol{u}_{0}\right\|_{\infty}\|\nabla \boldsymbol{v}\|_{2}+\left\|\nabla \boldsymbol{u}_{0}\right\|_{\frac{2 q}{2-q}}\|\boldsymbol{v}\|_{q^{\prime}}\right)
\end{aligned}
$$

Using the Sobolev embedding $W^{1,2}(\Omega) \subset L^{q^{\prime}}(\Omega)$ in the latter, we thus infer that

$$
\left\|D^{2} \boldsymbol{v}\right\|_{2}+|\omega|^{\frac{1}{2}}\|\nabla \boldsymbol{v}\|_{2}+|\omega|\|\boldsymbol{v}\|_{2} \leq m_{1}\|\boldsymbol{v}\|_{2}+m_{2}\|\nabla \boldsymbol{v}\|_{2}
$$

where

$$
m_{1}:=c_{1} \lambda_{0}\left\|\nabla \boldsymbol{u}_{0}\right\|_{\frac{2 q}{2-q}}, \quad m_{2}=c_{1} \lambda_{0}\left(\left\|\boldsymbol{u}_{0}\right\|_{\infty}+\left\|\nabla \boldsymbol{u}_{0}\right\|_{\frac{2 q}{2-q}}\right),
$$

and $c_{1}=c_{1}(\Omega)$, from which the desired property follows by choosing $\bar{\omega}:=$ $\max \left\{m_{1}, m_{2}^{2}, \lambda_{0}^{2}, 1\right\}$. 
Denote by $U\left(\lambda_{0}\right)$ a neighborhood of $\lambda_{0}$, and, for $q \in(1,6 / 5)$, let

$$
\lambda \in U\left(\lambda_{0}\right) \mapsto \boldsymbol{u}(\lambda) \in X^{2, q}(\Omega),
$$

be a continuous map with $\boldsymbol{u}\left(\lambda_{0}\right)=\boldsymbol{u}_{0}$. Consider, alongside, the oneparameter family of operators defined by

$$
\begin{aligned}
\mathscr{L}(\lambda): \boldsymbol{v} \in \mathrm{D} & (\mathscr{L}(\lambda)) \subset H_{\mathbb{C}}(\Omega) \\
& \mapsto \mathrm{P}\left[\Delta \boldsymbol{v}+\lambda\left(\partial_{1} \boldsymbol{v}-\boldsymbol{u}(\lambda) \cdot \nabla \boldsymbol{v}-\boldsymbol{v} \cdot \nabla \boldsymbol{u}(\lambda)\right)\right] \in H_{\mathbb{C}}(\Omega),
\end{aligned}
$$

with $\mathrm{D}(\mathscr{L}(\lambda)) \equiv \mathrm{D}\left(\mathscr{L}_{0}\right)=Z_{\mathbb{C}}^{2,2}(\Omega)$, and $\lambda \in U\left(\lambda_{0}\right)$. Obviously, $\mathscr{L}\left(\lambda_{0}\right)=$ $\mathscr{L}_{0}$. Assume, next, that

$$
\mu_{0}:=\mathrm{i} \omega_{0}, \quad \text { some } \omega_{0} \in \mathbb{R}-\{0\},
$$

is in the spectrum of $\mathscr{L}\left(\lambda_{0}\right)$. Then, by Proposition $3.3, \mu_{0}$ must be an eigenvalue of finite multiplicity. We are interested in the behavior of the eigenvalues, $\mu=\mu(\lambda)$, of $\mathscr{L}(\lambda)$ for $\lambda \in U\left(\lambda_{0}\right)$. To this end, we recall that $\mu_{0}$ is simple (of multiplicity 1 , that is) if, denoting by $\boldsymbol{v}_{0}$ the corresponding normalized eigenvector, $\boldsymbol{v}_{0} \notin \mathrm{R}\left[\mathscr{L}_{0}-\mu_{0} I\right]$. Since $\mathscr{L}_{0}-\mu_{0} I$ is Fredholm of index 0 , we have $\operatorname{dim} \mathrm{N}\left[\mathscr{L}_{0}-\mu_{0} I\right]=\operatorname{codim} \mathrm{R}\left[\mathscr{L}_{0}-\mu_{0} I\right]=1$, and this implies, in particular, that, letting $\mathscr{L}_{0}^{*}$ be the adjoint operator of $\mathscr{L}_{0}, \operatorname{dim} \mathrm{N}\left[\mathscr{L}_{0}^{*}-\mu_{0} I\right]=1$ and that there is $\boldsymbol{v}_{0}^{*} \in \mathrm{N}\left[\mathscr{L}_{0}^{*}-\mu_{0} I\right]$ such that $\left\langle\boldsymbol{v}_{0}^{*}, \boldsymbol{v}_{0}\right\rangle \neq 0$; see, e.g., [39, Section 8.4]. For convenience, we normalize $\boldsymbol{v}_{0}^{*}$ in such a way that

$$
\left\langle\boldsymbol{v}_{0}^{*}, \boldsymbol{v}_{0}\right\rangle=\pi^{-1} \text {. }
$$

The following result holds (see [40, Proposition 79.15 and Corollary 79.16]).

Proposition 3.4 Let $\mu_{0}$ be a simple eigenvalue of $\mathscr{L}_{0}$, and let the map (3.17) be of class $C^{k}, k \geq 1$. Then, there are neighborhoods $U_{1}\left(\lambda_{0}\right) \subseteq U\left(\lambda_{0}\right)$ of $\lambda_{0}$, and $V\left(\mu_{0}\right) \subset \mathbb{C}$ of $\mu_{0}$, such that for each $\lambda \in U_{1}\left(\lambda_{0}\right)$ there is one and only one eigenvalue $\mu(\lambda) \in V\left(\mu_{0}\right)$ of $\mathscr{L}(\lambda)$. Moreover, the map $\lambda \mapsto \mu(\lambda)$ is of class $C^{k}$ and we have

$\mu^{\prime}\left(\lambda_{0}\right)=\left\langle\boldsymbol{v}_{0}^{*}, \partial_{1} \boldsymbol{v}_{0}-\boldsymbol{u}_{0} \cdot \nabla \boldsymbol{v}_{0}-\boldsymbol{v}_{0} \cdot \nabla \boldsymbol{u}_{0}-\lambda_{0}\left(\boldsymbol{u}^{\prime}\left(\lambda_{0}\right) \cdot \nabla \boldsymbol{v}_{0}+\boldsymbol{v}_{0} \cdot \nabla \boldsymbol{u}^{\prime}\left(\lambda_{0}\right)\right)\right\rangle$.

We now turn our focus to the study of some important properties of the time-dependent operator

$$
\mathscr{Q}:=\omega_{0} \partial_{\tau}-\mathscr{L}_{0}: \mathscr{W}_{2 \pi, 0}^{2}(\Omega) \mapsto \mathscr{H}_{2 \pi, 0}(\Omega), \quad \omega_{0}>0 .
$$


In particular, we are interested in determining necessary and sufficient conditions under which $\mathscr{Q}$ possesses a bounded inverse. To this end, we begin to recall the following result, which is a particular case of that proved in [14, Proposition 3]

Lemma 3.4 The operator

$$
\omega_{0} \partial_{\tau}-\mathrm{P}\left[\Delta+\lambda_{0} \partial_{1}\right]: \mathscr{W}_{2 \pi, 0}^{2}(\Omega) \mapsto \mathscr{H}_{2 \pi, 0}(\Omega)
$$

is a homeomorphism.

With the help of this lemma, we can prove the following one.

Lemma 3.5 Let $\boldsymbol{u}_{0} \in X^{2, q}(\Omega)$. Then, the operator $\mathscr{Q}$ defined in (3.21) is Fredholm of index 0 .

Proof. In view of Lemma 3.4, it is enough to show that the operator

$$
\mathscr{C}: \boldsymbol{v} \in \mathscr{W}_{2 \pi, 0}^{2}(\Omega) \mapsto \boldsymbol{u}_{0} \cdot \nabla \boldsymbol{v}+\boldsymbol{v} \cdot \nabla \boldsymbol{u}_{0} \in \mathscr{L}_{2 \pi, 0}^{2}(\Omega)
$$

is compact. Let $\left\{\boldsymbol{v}_{k}\right\} \subset \mathscr{W}_{2 \pi, 0}^{2}(\Omega)$ with $\left\|\boldsymbol{v}_{k}\right\|_{\mathscr{W}_{2 \pi, 0}^{2}}=1$, for all $k \in \mathbb{N}$. We may then select a sequence (again denoted by $\left\{\boldsymbol{v}_{k}\right\}$ ) and find $\boldsymbol{v}_{*} \in \mathscr{W}_{2 \pi, 0}^{2}(\Omega)$ such that

$$
\boldsymbol{v}_{k} \rightarrow \boldsymbol{v}_{*} \text { weakly in } \mathscr{W}_{2 \pi, 0}^{2}(\Omega) .
$$

Without loss of generality, we may take $\boldsymbol{v}_{*} \equiv \mathbf{0}$. From (3.22) and LionsAubin lemma we then have

$$
\int_{0}^{2 \pi}\left(\left\|\boldsymbol{v}_{k}(\tau)\right\|_{2, \Omega_{R}}^{2}+\left\|\nabla \boldsymbol{v}_{k}(\tau)\right\|_{2, \Omega_{R}}^{2}\right) \rightarrow 0 \text { as } k \rightarrow \infty, \text { for all } R>R_{*},
$$

which implies, by embedding,

$$
\int_{0}^{2 \pi}\left\|\boldsymbol{v}_{k}(\tau)\right\|_{q^{\prime}, \Omega_{R}}^{2} \rightarrow 0 \text { as } k \rightarrow \infty, \text { for all } R>R_{*},
$$

By the Hölder inequality,

$$
\int_{0}^{2 \pi}\left\|\boldsymbol{u}_{0} \cdot \nabla \boldsymbol{v}_{k}(\tau)\right\|_{2}^{2} \leq\left\|\boldsymbol{u}_{0}\right\|_{\infty}^{2} \int_{0}^{2 \pi}\left\|\nabla \boldsymbol{v}_{k}(\tau)\right\|_{2, \Omega_{R}}^{2}+\left\|\boldsymbol{u}_{0}\right\|_{\infty, \Omega^{R}}^{2} \int_{0}^{2 \pi}\left\|\nabla \boldsymbol{v}_{k}(\tau)\right\|_{2}^{2},
$$

which, by (3.23) and the arbitrariness of $R$ implies

$$
\lim _{k \rightarrow \infty} \int_{0}^{2 \pi}\left\|\boldsymbol{u}_{0} \cdot \nabla \boldsymbol{v}_{k}(\tau)\right\|_{2}^{2}=0
$$


Likewise, again by Hölder inequality,

$$
\begin{aligned}
\int_{0}^{2 \pi}\left\|\boldsymbol{v}_{k}(\tau) \cdot \nabla \boldsymbol{u}_{0}\right\|_{2}^{2} \leq\left\|\nabla \boldsymbol{u}_{0}\right\|_{\frac{2 q}{2-q}}^{2} \int_{0}^{2 \pi}\left\|\boldsymbol{v}_{k}(\tau)\right\|_{q^{\prime}, \Omega_{R}}^{2} \\
+\left\|\nabla \boldsymbol{u}_{0}\right\|_{\frac{2 q}{2-q}, \Omega^{R}}^{2} \int_{0}^{2 \pi}\left\|\boldsymbol{v}_{k}(\tau)\right\|_{q^{\prime}}^{2} .
\end{aligned}
$$

Recalling that $\mathscr{W}_{2 \pi, 0}^{2}(\Omega) \subset L^{\infty}\left(0,2 \pi ; L^{s}(\Omega)\right)$, for all $s \in[2, \infty)$ (e.g. [14, Lemma $2(\mathrm{a})]$, from the latter inequality and (3.24) we deduce

$$
\lim _{k \rightarrow \infty} \int_{0}^{2 \pi}\left\|\boldsymbol{v}_{k}(\tau) \cdot \nabla \boldsymbol{u}_{0}\right\|_{2}^{2}=0
$$

Combining (3.25) and (3.26) we thus conclude

$$
\lim _{k \rightarrow \infty}\left\|\mathscr{C}\left(\boldsymbol{v}_{k}\right)\right\|_{L^{2}\left(\Omega_{2 \pi}\right)}=0
$$

which completes the proof of the lemma.

Lemma 3.6 Let $\mathscr{L}_{0}$ be as in Proposition 3.3. Assume $\mu_{0}:=\mathrm{i} \omega_{0} \in \sigma\left(\mathscr{L}_{0}\right)$ is a simple eigenvalue, while $\mu_{k}:=\mathrm{i} k \omega_{0} \notin \sigma\left(\mathscr{L}_{0}\right)$, whenever $k \in \mathbb{N}-\{0,1\}(7)$ Let $\boldsymbol{v}_{0}$ be the (unique) normalized eigenvector corresponding to $\mu_{0}$, and set

$$
\boldsymbol{v}_{1}=\Re\left[\boldsymbol{v}_{0} \mathrm{e}^{\mathrm{i} \tau}\right], \quad \boldsymbol{v}_{2}=\Im\left[\boldsymbol{v}_{0} \mathrm{e}^{\mathrm{i} \tau}\right] .
$$

Then

$$
\operatorname{dim} N[\mathscr{Q}]=2
$$

and $\left\{\boldsymbol{v}_{1}, \boldsymbol{v}_{2}\right\}$ is a basis in $\mathrm{N}[\mathscr{Q}]$.

Proof. It is clear that $\mathcal{S}:=\operatorname{span}\left\{\boldsymbol{v}_{1}, \boldsymbol{v}_{2}\right\} \subseteq \mathrm{N}[\mathscr{Q}]$. Conversely, take $\boldsymbol{w} \in$ $\mathrm{N}[\mathscr{Q}]$. We may expand $\boldsymbol{w}$ in Fourier series

$$
\boldsymbol{w}=\sum_{\ell=-\infty}^{\infty} \boldsymbol{w}_{\ell} \mathrm{e}^{\mathrm{i} \ell \tau} ; \boldsymbol{w}_{\ell}(x):=\frac{1}{2 \pi} \int_{0}^{2 \pi} \boldsymbol{w}(x, t) \mathrm{e}^{-\mathrm{i} \ell \tau} d t ; \boldsymbol{w}_{0}(x) \equiv \mathbf{0}
$$

Evidently, $\boldsymbol{w}_{\ell} \in Z_{\mathbb{C}}^{2,2}(\Omega) \equiv \mathrm{D}_{\mathbb{C}}\left(\mathscr{L}_{0}\right)$. From $\mathscr{Q}(\boldsymbol{w})=\mathbf{0}$ we then deduce

$$
\mathrm{i} \ell \omega_{0} \boldsymbol{w}_{\ell}-\mathscr{L}_{0}\left(\boldsymbol{w}_{\ell}\right)=\mathbf{0}, \boldsymbol{w}_{\ell} \in \mathrm{D}_{\mathbb{C}}\left(\mathscr{L}_{0}\right), \quad \ell \in \mathbb{Z},
$$

\footnotetext{
(7) Notice that, by Proposition 3.3 there could be only a finite number of such $\mu_{k}$.
} 
which, recalling that from Proposition 3.3 the $\mu_{k}$ 's can only be eigenvalues, by assumption and (3.28) 3 implies $\boldsymbol{w}_{\ell}=\mathbf{0}$ for all $\ell \in \mathbb{Z}-\{ \pm 1\}$. Thus $\boldsymbol{w} \in \mathcal{S}$, and the lemma is completely proved.

We are now in a position to show the second main result of this section.

Proposition 3.5 Let the assumptions of Lemma 3.6 be satisfied, and set

$$
\boldsymbol{v}_{1}^{*}=\Re\left[\boldsymbol{v}_{0}^{*} \mathrm{e}^{-\mathrm{i} \tau}\right], \quad \boldsymbol{v}_{2}^{*}=\Im\left[\boldsymbol{v}_{0}^{*} \mathrm{e}^{-\mathrm{i} \tau}\right] .
$$

where $\boldsymbol{v}_{0}^{*}$ is the (uniquely determined) element of $\mathrm{N}\left[\mathscr{L}_{0}^{*}-\mu_{0} I\right]$ satisfying (3.19) Then, for a given $\boldsymbol{f} \in \mathscr{H}_{2 \pi, 0}(\Omega)$, necessary and sufficient condition for the problem

$$
\mathscr{Q}(\boldsymbol{v}):=\omega_{0} \partial_{\tau} \boldsymbol{v}-\mathscr{L}_{0}(\boldsymbol{v})=\boldsymbol{f}, \quad \boldsymbol{v} \in \mathscr{W}_{2 \pi, 0}^{2}(\Omega),
$$

to have a solution is that

$$
\left(\boldsymbol{v}_{1}^{*} \mid \boldsymbol{f}\right)=\left(\boldsymbol{v}_{2}^{*} \mid \boldsymbol{f}\right)=0 .
$$

This solution is also unique, provided $\left(\boldsymbol{v}_{1}^{*} \mid \boldsymbol{v}\right)=\left(\boldsymbol{v}_{2}^{*} \mid \boldsymbol{v}\right)=0$ and, in such a case, there is $c=c(\Omega)$ such that

$$
\|\boldsymbol{v}\|_{\mathscr{W}_{2 \pi, 0}^{2}} \leq c\|\boldsymbol{f}\|_{\mathscr{H}_{2 \pi, 0}} .
$$

Proof. Since, by Lemma 3.5, $\mathscr{Q}$ is Fredholm of index 0, and, by Lemma 3.6 $\operatorname{dim} N[\mathscr{Q}]=2$, it follows (e.g. [39, Proposition 8.14(4)]) that $\operatorname{dim} N\left[\mathscr{Q}^{*}\right]=2$ where

$$
\mathscr{Q}^{*}:=-\omega_{0} \partial_{\tau}-\mathscr{L}_{0}^{*}
$$

is the adjoint of $\mathscr{Q}$. In view of the stated properties of $\boldsymbol{v}_{0}^{*}$, we infer that $\operatorname{span}\left\{\boldsymbol{v}_{1}^{*}, \boldsymbol{v}_{2}^{*}\right\}=\mathrm{N}\left[\mathscr{Q}^{*}\right]$, and the proposition follows from another classical result on Fredholm operators (e.g., [39, Proposition 8.14(2)]).

\footnotetext{
${ }^{(8)}$ Recall that $\operatorname{dim} \mathrm{N}\left[\mathscr{L}_{0}^{*}-\mu_{0} I\right]=1$.
} 


\section{Bifurcating Time-Periodic Solutions.}

We begin to put the original problem (1.3)-(1.4) in a different and equivalent form that will allow us to employ the results established in the previous section.

To this end, let $\lambda_{0}(>0)$ be a value of the Reynolds number for which the steady-state problem (3.4) has a solution $\left(\boldsymbol{u}_{0}, p_{0}\right) \in X^{2, q}(\Omega) \times D^{1, q}(\Omega)$, $1<q<6 / 5$. We suppose that $\lambda_{0}$ is such that $\left(\boldsymbol{u}_{0}, p_{0}\right)$ is a point of an analytic solution branch $(\boldsymbol{u}(\lambda), p(\lambda))$ to (3.4), for all $\lambda$ in a neighborhood $U\left(\lambda_{0}\right)$ of $\lambda_{0}$. By Proposition 3.2 , such a $\lambda_{0}$ exists if we assume that

$$
\mathrm{N}\left[\widetilde{\mathscr{L}_{0}}\right]=\{0\}, \text { with } \widetilde{\mathscr{L}_{0}} \text { defined in }(\overline{3.2}),
$$

or, equivalently,

$$
\begin{gathered}
\left.\begin{array}{c}
\Delta \boldsymbol{u}+\lambda_{0}\left(\partial_{1} \boldsymbol{v}-\boldsymbol{u}_{0} \cdot \nabla \boldsymbol{u}-\boldsymbol{u} \cdot \nabla \boldsymbol{u}_{0}\right)=\nabla \phi \\
\operatorname{div} \boldsymbol{u}=0
\end{array}\right\} \text { in } \Omega \\
\boldsymbol{u}=\mathbf{0} \text { at } \partial \Omega, \quad(\boldsymbol{u}, \phi) \in X^{2, q}(\Omega) \times D^{1, q}(\Omega), 1<q<\frac{6}{5}
\end{gathered}
$$

Our first objective is to prove the existence of a family of time-periodic solutions of period $T:=2 \pi / \omega$ (to be determined) to (1.3), bifurcating from the point $\left(\lambda_{0} ;\left(\boldsymbol{u}_{0}, p_{0}\right)\right)$. To this end, following Lindtstedt [29] and Poincaré [35, we introduce the scaled time $\tau:=\omega t$, so that (1.3) becomes

$$
\left.\begin{array}{c}
\omega \partial_{\tau} \boldsymbol{v}+\lambda\left[\left(\boldsymbol{v}-\boldsymbol{e}_{1}\right) \cdot \nabla \boldsymbol{v}+\boldsymbol{u}(\lambda) \cdot \nabla \boldsymbol{v}+\boldsymbol{v} \cdot \nabla \boldsymbol{u}(\lambda)\right]=\Delta \boldsymbol{v}-\nabla \mathrm{p} \\
\operatorname{div} \boldsymbol{v}=0
\end{array}\right\} \text { in } \Omega_{2 \pi}
$$

We next split $\boldsymbol{v}$ and $\mathrm{p}$ as the sum of their time average, $(\overline{\boldsymbol{v}}, \overline{\mathrm{p}})$, over the time interval $[0,2 / \pi]$, and their "purely periodic" component $(\boldsymbol{w}:=$ $\boldsymbol{v}-\overline{\boldsymbol{v}}, \varphi:=\overline{\mathrm{p}}-\mathrm{p}$ ). In this way, problem (4.1) can be equivalently rewritten as the following coupled nonlinear elliptic-parabolic problem

$$
\left.\begin{array}{c}
\Delta \overline{\boldsymbol{v}}+\lambda_{0}\left(\partial_{1} \overline{\boldsymbol{v}}-\boldsymbol{u}_{0} \cdot \nabla \overline{\boldsymbol{v}}-\boldsymbol{u}_{0} \cdot \nabla \overline{\boldsymbol{v}}\right)=\nabla \overline{\mathbf{p}}+\boldsymbol{N}_{1}(\lambda, \overline{\boldsymbol{v}}, \boldsymbol{w}) \\
\operatorname{div} \overline{\boldsymbol{v}}=0 \\
\overline{\boldsymbol{v}}=\mathbf{0} \text { at } \partial \Omega
\end{array}\right\} \text { in } \Omega
$$


and

$$
\left.\begin{array}{rl}
\omega_{0} \partial_{\tau} \boldsymbol{w}-\Delta \boldsymbol{w}-\lambda_{0}\left(\partial_{1} \boldsymbol{w}-\boldsymbol{u}_{0} \cdot \nabla \boldsymbol{w}-\boldsymbol{w} \cdot \nabla \boldsymbol{u}_{0}\right) \\
& =\nabla \varphi+\boldsymbol{N}_{2}(\lambda, \omega, \overline{\boldsymbol{v}}, \boldsymbol{w}) \\
\operatorname{div} \boldsymbol{w} & =0 \\
\boldsymbol{w} & =\mathbf{0} \text { at } \partial \Omega_{2 \pi},
\end{array}\right\} \text { in } \Omega_{2 \pi}
$$

where $\omega_{0}>0$, and

$$
\begin{aligned}
\boldsymbol{N}_{1}:= & \left(\lambda_{0}-\lambda\right)\left[\partial_{1} \overline{\boldsymbol{v}}-\boldsymbol{u}(\lambda) \cdot \nabla \overline{\boldsymbol{v}}-\overline{\boldsymbol{v}} \cdot \nabla \boldsymbol{u}(\lambda)\right] \\
& +\lambda_{0}\left[\left(\boldsymbol{u}(\lambda)-\boldsymbol{u}_{0}\right) \cdot \nabla \overline{\boldsymbol{v}}+\overline{\boldsymbol{v}} \cdot \nabla\left(\boldsymbol{u}(\lambda)-\boldsymbol{u}_{0}\right)\right] \\
& +\lambda[\overline{\boldsymbol{v}} \cdot \nabla \overline{\boldsymbol{v}}+\overline{\boldsymbol{w} \cdot \nabla \boldsymbol{w}}]
\end{aligned}
$$

and

$$
\begin{aligned}
\boldsymbol{N}_{2}:= & \left(\omega_{0}-\omega\right) \partial_{\tau} \boldsymbol{w}+\left(\lambda-\lambda_{0}\right)\left[\partial_{1} \boldsymbol{w}-\boldsymbol{u}(\lambda) \cdot \nabla \boldsymbol{w}-\boldsymbol{w} \cdot \nabla \boldsymbol{u}(\lambda)\right] \\
& -\lambda_{0}\left[\left(\boldsymbol{u}(\lambda)-\boldsymbol{u}_{0}\right) \cdot \nabla \boldsymbol{w}+\boldsymbol{w} \cdot \nabla\left(\boldsymbol{u}(\lambda)-\boldsymbol{u}_{0}\right)\right] \\
& +\lambda[\boldsymbol{w} \cdot \nabla \overline{\boldsymbol{v}}+\overline{\boldsymbol{v}} \cdot \nabla \boldsymbol{w}+\boldsymbol{w} \cdot \nabla \boldsymbol{w}-\overline{\boldsymbol{w} \cdot \nabla \boldsymbol{w}}]
\end{aligned}
$$

Some functional properties of the quantities $\boldsymbol{N}_{i}, i=1,2$, are proved next.

Lemma 4.1 Let $1<q<6 / 5$. The following bilinear maps are continuous

$$
\begin{aligned}
& \mathcal{M}_{1}:\left(\boldsymbol{v}_{1}, \boldsymbol{v}_{2}\right) \in\left[X^{2, q}(\Omega)\right]^{2} \mapsto \boldsymbol{v}_{1} \cdot \nabla \boldsymbol{v}_{2} \in L^{q}(\Omega), \\
& \mathcal{M}_{2}:\left(\boldsymbol{w}_{1}, \boldsymbol{w}_{2}\right) \in\left[\mathscr{W}_{2 \pi, 0}^{2}(\Omega)\right]^{2} \mapsto \int_{0}^{2 \pi} \boldsymbol{w}_{1} \cdot \nabla \boldsymbol{w}_{2} \in L^{r}(\Omega), r=q, 2, \\
& \mathcal{M}_{3}:(\boldsymbol{v}, \boldsymbol{w}) \in X^{2, q}(\Omega) \times \mathscr{W}_{2 \pi, 0}^{2}(\Omega) \mapsto \boldsymbol{v} \cdot \nabla \boldsymbol{w} \in \mathscr{L}_{2 \pi, 0}^{2}(\Omega), \\
& \mathcal{M}_{4}:(\boldsymbol{v}, \boldsymbol{w}) \in X^{2, q}(\Omega) \times \mathscr{W}_{2 \pi, 0}^{2}(\Omega) \mapsto \boldsymbol{w} \cdot \nabla \boldsymbol{v} \in \mathscr{L}_{2 \pi, 0}^{2}(\Omega), \\
& \mathcal{M}_{5}:\left(\boldsymbol{w}_{1}, \boldsymbol{w}_{2}\right) \in\left[\mathscr{W}_{2 \pi, 0}^{2}(\Omega)\right]^{2} \mapsto \boldsymbol{w}_{1} \cdot \nabla \boldsymbol{w}_{2} \in \mathscr{L}_{2 \pi, 0}^{2}(\Omega) .
\end{aligned}
$$

Proof. The continuity of $\mathcal{M}_{1}$ is shown in [12, Lemma XII.5.4]. In order to show the remaining properties, we recall the following continuous embeddings (see [14, Lemmas 1 and 2])

$$
\begin{gathered}
X^{2, q}(\Omega) \subset L^{\infty}(\Omega) ; \quad X^{2, q}(\Omega) \subset D^{1, \frac{2 q}{2-q}}(\Omega) ; \\
\mathscr{W}_{2 \pi, 0}^{2}(\Omega) \subset L^{\infty}\left(0,2 \pi ; L^{s}(\Omega)\right) \text {, all } s \in[2, \infty) ; \mathscr{W}_{2 \pi, 0}^{2}(\Omega) \subset L^{4}\left(0,2 \pi ; W^{1,4}(\Omega)\right) .
\end{gathered}
$$


Therefore, by (4.6) and Hölder inequality we deduce

$$
\begin{aligned}
& \left\|\mathcal{M}_{2}\left(\boldsymbol{w}_{1}, \boldsymbol{w}_{2}\right)\right\|_{q} \leq \int_{0}^{2 \pi}\left\|\boldsymbol{w}_{1}\right\|_{\frac{2 q}{2-q}}\left\|\nabla \boldsymbol{w}_{2}\right\|_{2} \leq c_{1}\left\|\boldsymbol{w}_{1}\right\|_{\mathscr{W}_{2 \pi, 0}^{2}}\left\|\boldsymbol{w}_{2}\right\|_{\mathscr{W}_{2 \pi, 0}^{2}} \\
& \left\|\mathcal{M}_{2}\left(\boldsymbol{w}_{1}, \boldsymbol{w}_{2}\right)\right\|_{2} \leq \int_{0}^{2 \pi}\left\|\boldsymbol{w}_{1}\right\|_{4}\left\|\nabla \boldsymbol{w}_{2}\right\|_{4} \leq c_{2}\left\|\boldsymbol{w}_{1}\right\|_{\mathscr{W}_{2 \pi, 0}^{2}}\left\|\boldsymbol{w}_{2}\right\|_{\mathscr{W}_{2 \pi, 0}^{2}} \\
& \left\|\mathcal{M}_{3}(\boldsymbol{w}, \boldsymbol{w})\right\|_{\mathscr{L}_{2 \pi, 0}^{2}} \leq\|\boldsymbol{v}\|_{\infty}\left(\int_{0}^{2 \pi}\left\|\nabla \boldsymbol{w}_{2}\right\|_{2}^{2}\right)^{\frac{1}{2}} \leq c_{3}\|\boldsymbol{v}\|_{X^{2, q}}\left\|\boldsymbol{w}_{2}\right\|_{\mathscr{W}_{2 \pi, 0}^{2}} \\
& \left\|\mathcal{M}_{4}(\boldsymbol{w}, \boldsymbol{v})\right\|_{2} \leq\|\nabla \boldsymbol{v}\|_{\frac{2 q}{2-q}}\left(\int_{0}^{2 \pi}\|\boldsymbol{w}\|_{q^{\prime}}^{2}\right)^{\frac{1}{2}} \leq c_{4}\|\boldsymbol{v}\|_{X^{2, q}}\|\boldsymbol{w}\|_{\mathscr{W}_{2 \pi, 0}^{2}} \\
& \left\|\mathcal{M}_{5}\left(\boldsymbol{w}_{1}, \boldsymbol{w}_{2}\right)\right\|_{2} \leq\left(\int_{0}^{2 \pi}\left\|\boldsymbol{w}_{1}\right\|_{4}^{4}\right)^{\frac{1}{4}}\left(\int_{0}^{2 \pi}\left\|\nabla \boldsymbol{w}_{2}\right\|_{4}^{4}\right)^{\frac{1}{4}} \leq c_{5}\left\|\boldsymbol{w}_{1}\right\|_{\mathscr{W}_{2 \pi, 0}^{2}}\left\|\boldsymbol{w}_{2}\right\|_{\mathscr{W}_{2 \pi, 0}^{2}}
\end{aligned}
$$

With the help of this lemma, and recalling the definition of $\widetilde{\mathscr{L}}_{0}$ and $\mathscr{Q}$ given in (3.4) and (3.23), respectively, we may infer that problems (4.2)-(4.5) can be equivalently rewritten in the following operator form

$$
\begin{aligned}
& \widetilde{\mathscr{L}_{0}}(\overline{\boldsymbol{v}})=\mathcal{N}_{1}(\lambda, \overline{\boldsymbol{v}}, \boldsymbol{w}) \text { in } H_{q}(\Omega), \\
& \mathscr{Q}(\boldsymbol{w})=\mathcal{N}_{2}(\lambda, \omega, \overline{\boldsymbol{v}}, \boldsymbol{w}) \text { in } \mathscr{H}_{2 \pi, 0}(\Omega),
\end{aligned}
$$

where $\mathcal{N}_{i}=\mathrm{P} \boldsymbol{N}_{i}, i=1,2$.

The desired bifurcation result will be obtained by showing that, under appropriate assumptions on $\left(\lambda_{0}, \omega_{0}\right)$, there exists a non-trivial family of solutions $(\overline{\boldsymbol{v}}, \boldsymbol{w}) \in X_{0}^{2, q}(\Omega) \times \mathscr{W}_{2 \pi, 0}^{2}(\Omega)$ to (4.8) for $(\lambda, \omega)$ in a neighborhood of $\left(\lambda_{0}, \omega_{0}\right)$.

Remark 4.1 The asymptotic side condition (1.4) is embodied in the function spaces where $\overline{\boldsymbol{v}}$ and $\boldsymbol{w}$ are sought. In fact, since $\overline{\boldsymbol{v}} \in X_{0}^{2, q}(\Omega)$, from [14, Lemma 1] we have

$$
\lim _{|x| \rightarrow \infty}|\overline{\boldsymbol{v}}(x)|=0 \text { uniformly, }
$$

whereas $\boldsymbol{w} \in \mathscr{W}_{2 \pi, 0}^{2}(\Omega)$ and [12, Theorem II.9.1] imply, for almost all $t \in$ $[0,2 \pi]$,

$$
\lim _{|x| \rightarrow \infty}|\boldsymbol{w}(x, t)|=0 \quad \text { uniformly in } x .
$$

We next recall that, by Proposition 3.3, if i $\omega_{0} \in \sigma\left(\mathscr{L}_{0}\right)$ then it must be an eigenvalue of finite multiplicity, and, moreover, there is at most, a finite 
number of eigenvalues of the form i $k \omega_{0}$ with $k \in \mathbb{N}$. With this in mind, we make the more stringent hypothesis that $\omega_{0}$ be such that

$$
\begin{aligned}
& \mu_{0}:=\mathrm{i} \omega_{0} \text { is an eigenvalue of multiplicity } 1 \text { of } \mathscr{L}_{0}, \\
& k \mu_{0}, k \in \mathbb{N}-\{0,1\} \text { is not an eigenvalue of } \mathscr{L}_{0},
\end{aligned}
$$

and look for solutions to (4.8) satisfying the further requirement

$$
\left(\boldsymbol{w} \mid \boldsymbol{v}_{1}^{*}\right)=\varepsilon, \quad\left(\boldsymbol{w} \mid \boldsymbol{v}_{2}^{*}\right)=0,
$$

where $\boldsymbol{v}_{i}^{*}, i=1,2$, is defined in Proposition 3.5. and $\varepsilon \in(-1,1)$.

We are now in a position to prove our main result on the existence and uniqueness of bifurcating time-periodic solutions, along with their relevant properties. To this end, we observe that, under the assumptions ([H1) and (1H2) the eigenvalue $\mu(\lambda)$ of the operator $\mathscr{L}(\lambda)$ defined in (3.21) is a $C^{\infty}$. function of $\lambda$ in a suitable neighborhood of $\lambda_{0}$, and (3.20) holds.

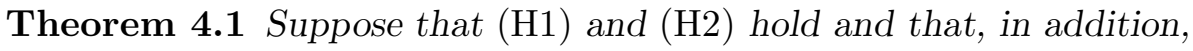

$$
\Re\left[\mu^{\prime}\left(\lambda_{0}\right)\right] \neq 0 .
$$

Then, the following properties are valid.

(a) Existence. There are (real) analytic families

$$
(\overline{\boldsymbol{v}}(\varepsilon), \boldsymbol{w}(\varepsilon), \omega(\varepsilon), \lambda(\varepsilon)) \in X_{0}^{2, q}(\Omega) \times \mathscr{W}_{2 \pi, 0}^{2}(\Omega) \times \mathbb{R}_{+}^{2}
$$

satisfying (4.8)-(4.8) for all $\varepsilon$ in a neighborhood $\mathcal{I}(0)$ of 0 , and such that

$$
\left(\overline{\boldsymbol{v}}(\varepsilon), \boldsymbol{w}(\varepsilon)-\varepsilon \boldsymbol{v}_{1}, \omega(\varepsilon), \lambda(\varepsilon)\right) \rightarrow\left(\mathbf{0}, \mathbf{0}, \omega_{0}, \lambda_{0}\right) \text { as } \varepsilon \rightarrow 0
$$

with $\boldsymbol{v}_{1}$ given in (3.27) . Moreover, the corresponding velocity field $\boldsymbol{V}$ of the original problem (1.3) has the following form near $\varepsilon=0$

$$
\boldsymbol{V}(x, \tau ; \lambda(\varepsilon))=\boldsymbol{u}_{0}(\boldsymbol{x})+\varepsilon\left[(\cos \tau) \boldsymbol{a}_{1}+(\sin \tau) \boldsymbol{a}_{2}\right]+\varepsilon^{2}\left[\boldsymbol{V}_{1}+\boldsymbol{V}_{2}\right],
$$

where $\boldsymbol{a}_{i} \in Z^{2,2}(\Omega), i=1,2$, and $\left(\boldsymbol{V}_{1}, \boldsymbol{V}_{2}\right) \in X_{0}^{2, q}(\Omega) \times \mathscr{W}_{2 \pi, 0}^{2}(\Omega)$ satisfy

$$
\left\|\boldsymbol{V}_{1}\right\|_{X^{2 q}}+\left\|\boldsymbol{V}_{2}\right\|_{\mathscr{W}_{2 \pi, 0}^{2}} \leq M
$$

with $M$ independent of $\varepsilon \rightarrow 0$. 
(b) Uniqueness. There is a neighborhood

$$
\mathscr{U}\left(\mathbf{0}, \mathbf{0}, \omega_{0}, \lambda_{0}\right) \subset X_{0}^{2, q}(\Omega) \times \mathscr{W}_{2 \pi, 0}^{2}(\Omega) \times \mathbb{R}_{+}^{2}
$$

such that every (nontrivial) solution to (4.8) lying in $\mathscr{U}$ must belong, up to a phase shift, to the family (4.12).

(c) Parity. The functions $\omega(\varepsilon)$ and $\lambda(\varepsilon)$ are even:

$$
\omega(\varepsilon)=\omega(-\varepsilon), \quad \lambda(\varepsilon)=\lambda(-\varepsilon), \text { for all } \varepsilon \in \mathcal{I}(0) .
$$

Consequently, the bifurcation due to these solutions is either subcritical or supercritical, a two-sided bifurcation being excluded (9)

Proof. We rescale our original unknowns in (4.8)-(4.8) as follows:

$$
\boldsymbol{w}=\varepsilon \mathrm{W}, \quad \overline{\boldsymbol{v}}=\varepsilon \mathrm{v},
$$

so that (4.8)-(4.8) can be equivalently written as

$$
\begin{aligned}
& \widetilde{\mathscr{L}}_{0}(\mathrm{v})-\mathscr{N}_{1}(\varepsilon, \lambda, \mathrm{v}, \mathrm{w})=0 \text { in } H_{q}(\Omega), \\
& \mathscr{Q}(\mathrm{w})-\mathscr{N}_{2}(\varepsilon, \lambda, \omega, \mathrm{v}, \mathrm{w})=0 \text { in } \mathscr{L}_{2 \pi, 0}^{2}(\Omega), \\
& \left(\mathrm{w} \mid \boldsymbol{v}_{1}^{*}\right)=1, \quad\left(\mathrm{w} \mid \boldsymbol{v}_{2}^{*}\right)=0,
\end{aligned}
$$

where

$$
\begin{aligned}
\mathscr{N}_{1}:= & \mathrm{P}\left\{\left(\lambda_{0}-\lambda\right)\left[\partial_{1} \mathbf{v}-\boldsymbol{u}(\lambda) \cdot \nabla \mathrm{v}-\mathrm{v} \cdot \nabla \boldsymbol{u}(\lambda)\right]\right. \\
& +\lambda_{0}\left[\left(\boldsymbol{u}(\lambda)-\boldsymbol{u}_{0}\right) \cdot \nabla \mathrm{v}+\mathrm{v} \cdot \nabla\left(\boldsymbol{u}(\lambda)-\boldsymbol{u}_{0}\right)\right] \\
& +\lambda \varepsilon[\mathrm{v} \cdot \nabla \mathrm{v}+\overline{\mathrm{w} \cdot \nabla \mathrm{w}}]\},
\end{aligned}
$$

and

$$
\begin{aligned}
\mathscr{N}_{2}:= & \mathrm{P}\left\{\left(\omega_{0}-\omega\right) \partial_{t} \mathrm{w}+\left(\lambda-\lambda_{0}\right)\left[\partial_{1} \mathrm{w}-\boldsymbol{u}(\lambda) \cdot \nabla \mathrm{w}-\mathrm{w} \cdot \nabla \boldsymbol{u}(\lambda)\right]\right. \\
& -\lambda_{0}\left[\left(\boldsymbol{u}(\lambda)-\boldsymbol{u}_{0}\right) \cdot \nabla \mathrm{w}+\mathrm{w} \cdot \nabla\left(\boldsymbol{u}(\lambda)-\boldsymbol{u}_{0}\right)\right] \\
& +\lambda \varepsilon[\mathrm{w} \cdot \nabla \mathrm{v}+\mathrm{v} \cdot \nabla \mathrm{w}+\mathrm{w} \cdot \nabla \mathrm{w}-\overline{\mathrm{w} \cdot \nabla \mathrm{w}}]\}
\end{aligned}
$$

Define the map:

$$
\begin{gathered}
F:(\varepsilon, \lambda, \omega, \mathrm{v}, \mathrm{w}) \in \mathcal{I}(0) \times U\left(\lambda_{0}\right) \times V\left(\omega_{0}\right) \times X_{0}^{2, q}(\Omega) \times \mathscr{W}_{2 \pi, 0}^{2}(\Omega) \\
\mapsto\left(\widetilde{\mathscr{L}}_{0}(\mathrm{v})-\mathscr{N}_{1}(\varepsilon, \lambda, \mathrm{v}, \mathrm{w}), \mathscr{Q}(\mathrm{w})-\mathscr{N}_{2}(\varepsilon, \lambda, \omega, \mathrm{v}, \mathrm{w}),\left(\mathrm{w} \mid \boldsymbol{v}_{1}^{*}\right)-1,\left(\mathrm{w} \mid \boldsymbol{v}_{2}^{*}\right)\right) \\
\in H_{q}(\Omega) \times \mathscr{H}_{2 \pi, 0}(\Omega) \times \mathbb{R}^{2} .
\end{gathered}
$$

\footnotetext{
${ }^{(9)}$ Unless $\lambda \equiv \lambda_{0}$.
} 
The nonlinear terms in (4.17) -(4.18) are of polynomial form, and, by (H1) and Proposition 3.2, $\boldsymbol{u}(\lambda)$ is analytic. Thus, also with the help of Lemma 4.1 we may conclude that $F$ is analytic. Furthermore, from (4.16)-(4.18) and (H1) it follows that for $\varepsilon=0$, the equation $F=0$ has the solution $\left(\lambda=\lambda_{0}, \omega=\omega_{0}, v=\mathbf{0}, \mathbf{w}=\boldsymbol{v}_{1}\right)$. Therefore, by the (real) analytic version of the implicit function theorem (e.g. [39, Proposition 8.11]), to show the existence part in the theorem -including the validity of (4.13)- it suffices to show that the Fréchet derivative of $F$ with respect to $U:=(\lambda, \omega, \mathrm{v}, \mathrm{w})$ evaluated at $\left(\varepsilon=0, \lambda=\lambda_{0}, \omega=\omega_{0}, \mathbf{v}=\mathbf{0}, \mathbf{w}=\boldsymbol{v}_{1}\right)$ is a bijection. The latter will hold if we prove that for any $\left(\mathrm{f}_{1}, \mathrm{f}_{2}, \mathrm{f}_{3}, \mathrm{f}_{4}\right) \in H_{q}(\Omega) \times \mathscr{H}_{2, \pi, 0}(\Omega) \times \mathbb{R} \times \mathbb{R}$, the following set of equations has one and only one solution $(\lambda, \omega, \mathrm{v}, \mathrm{w}) \in$ $\mathbb{R} \times \mathbb{R} \times X_{0}^{2, q}(\Omega) \times \mathscr{W}_{2 \pi, 0}^{2}(\Omega):$

$$
\begin{aligned}
\widetilde{\mathscr{L}_{0}}(\mathrm{v}) & =\mathrm{f}_{1} \text { in } H_{q}(\Omega) \\
\mathscr{Q}(\mathrm{w}) & =\mathscr{F}\left(\lambda, \omega, \boldsymbol{v}_{1}\right)+\mathrm{f}_{2} \text { in } \mathscr{H}_{2 \pi, 0}(\Omega), \\
\left(\mathrm{w} \mid \boldsymbol{v}_{1}^{*}\right) & =\mathrm{f}_{3}, \quad\left(\mathrm{w} \mid \boldsymbol{v}_{2}^{*}\right)=\mathrm{f}_{4} \text { in } \mathbb{R},
\end{aligned}
$$

where

$$
\begin{array}{r}
\mathscr{F}\left(\lambda, \omega, \boldsymbol{v}_{1}\right):=-\omega \partial_{\tau} \boldsymbol{v}_{1}+\lambda \mathrm{P}\left\{\partial_{1} \boldsymbol{v}_{1}-\boldsymbol{u}_{0} \cdot \nabla \boldsymbol{v}_{1}-\boldsymbol{v}_{1} \cdot \nabla \boldsymbol{u}_{0}\right. \\
\left.-\lambda_{0}\left(\boldsymbol{u}^{\prime}\left(\lambda_{0}\right) \cdot \nabla \boldsymbol{v}_{1}+\boldsymbol{v}_{1} \cdot \nabla \boldsymbol{u}^{\prime}\left(\lambda_{0}\right)\right)\right\} .
\end{array}
$$

Since $\widetilde{\mathscr{L}_{0}}$ is Fredholm of index 0 (Proposition [3.1), in view of (ㅍ1), for any given $f_{1} \in H_{q}(\Omega)$, equation (4.19) 1 has one and only one solution $\mathrm{v} \in X^{2, q}(\Omega)$. Therefore, it remains to prove the existence and uniqueness property only for the system of equations $(4.19)_{2-4}$ To this aim, we observe that, by Proposition 3.5, for a given $\mathrm{f}_{2} \in \mathscr{H}_{2 \pi, 0}(\Omega)$, equation (4.19) 2 possesses a solution $w \in \mathscr{W}_{2 \pi, 0}^{2}(\Omega)$ if and only if its right-hand side satisfies (3.30). By a direct calculation, from (3.27), (3.29), and (3.19) we show

$$
\left(\partial_{\tau} \boldsymbol{v}_{1} \mid \boldsymbol{v}_{1}^{*}\right)=0, \quad\left(\partial_{\tau} \boldsymbol{v}_{1} \mid \boldsymbol{v}_{2}^{*}\right)=\pi
$$

Furthermore, again by a straightforward calculation that uses also (3.20) and the fact that $\mathrm{P}$ is self-adjoint in $L^{2}$ and $\mathrm{P} \boldsymbol{v}_{1}^{*}=\boldsymbol{v}_{1}^{*}$, we infer

$$
\begin{array}{r}
\left\langle\mathrm{P}\left\{\partial_{1} \boldsymbol{v}_{1}-\boldsymbol{u}_{0} \cdot \nabla \boldsymbol{v}_{1}-\boldsymbol{v}_{1} \cdot \nabla \boldsymbol{u}_{0}+\lambda_{0}\left(\boldsymbol{u}^{\prime}\left(\lambda_{0}\right) \cdot \nabla \boldsymbol{v}_{1}+\boldsymbol{v}_{1} \cdot \nabla \boldsymbol{u}^{\prime}\left(\lambda_{0}\right)\right)\right\}, \boldsymbol{v}_{1}^{*}\right\rangle \\
=\Re\left[\mu^{\prime}\left(\lambda_{0}\right)\right] .
\end{array}
$$


Employing (4.21) and (4.22), we thus recognize that the compatibility condition (3.30) for the solvability of equation (4.19) 2 reduces to solving the following algebraic system for $\lambda$ and $\omega$ :

$$
\begin{aligned}
\lambda \Re\left[\mu^{\prime}\left(\lambda_{0}\right)\right] & =-\left(\mathrm{f}_{2} \mid \boldsymbol{v}_{1}^{*}\right) \\
-\omega \pi+\lambda\left(\mathscr{F} \mid \boldsymbol{v}_{2}^{*}\right) & =-\left(\mathrm{f}_{2} \mid \boldsymbol{v}_{2}^{*}\right),
\end{aligned}
$$

with $\mathscr{F}$ given in (4.20). By virtue of ( $(\mathrm{H3})$, for any given $\mathrm{f}_{2}$ in the specified class, we can always find (uniquely determined) $\lambda$ and $\omega$ satisfying the above system, and this, by Proposition 3.5. ensures the existence of a solution $w_{1}$ to (4.19) 2 corresponding to the selected values of $\lambda$ and $\omega$. We now set

$$
\mathrm{w}:=\mathrm{w}_{1}+\alpha \boldsymbol{v}_{1}+\beta \boldsymbol{v}_{2}, \quad \alpha, \beta \in \mathbb{R} .
$$

Clearly, by Lemma 3.6, $\mathrm{w}$ is also a solution to (4.19) 2 . We then choose $\alpha$ and $\beta$ in such a way that $w$ satisfies both conditions $(4.19){ }_{3,4}$ for any given $f_{i} \in \mathbb{R}$, $i=1,2$. This choice is made possible by the fact that, as is immediately checked,

$$
\left(\boldsymbol{v}_{i} \mid \boldsymbol{v}_{j}^{*}\right)=\delta_{i j}, \quad i, j=1,2 .
$$

The existence part is therefore accomplished. We now turn to uniqueness and set $\mathrm{f}_{i}=\mathbf{0}$ in (4.19) $)_{2-4}$. From (4.23) and (플 $)$ it then follows $\lambda=\omega=0$ which in turn implies, by (4.19) 2 and Lemma 3.6. $\mathbf{w}=\gamma_{1} \boldsymbol{v}_{1}+\gamma_{2} \boldsymbol{v}_{2}$, for some $\gamma_{i} \in \mathbb{R}, i=1,2$. Replacing this information back in (4.19) 3,4 with $\mathrm{f}_{3}=\mathrm{f}_{4}=\mathbf{0}$, and using (4.24) we conclude $\gamma_{1}=\gamma_{2}=0$, which completes the uniqueness proof. We have thus shown that the above specified Fréchet derivative of $F$ is a bijection, which ensures existence to (4.16)-(4.18), and therefore of a family of solutions, parametrized in $\varepsilon$, to (4.8)-(4.8) in the sense specified in (a). To complete the proof of the statement in (a), it remains to show (4.14). To this end, we begin to notice that from (3.27) we have

$$
\boldsymbol{v}_{1}=(\cos \tau) \boldsymbol{a}_{1}+(\sin \tau) \boldsymbol{a}_{2}, \quad \boldsymbol{a}_{1}, \boldsymbol{a}_{2} \in Z^{2,2}(\Omega) .
$$

Next, let us give for granted, momentarily, the result in (c). By the analyticity property of $\lambda(\varepsilon)$ we then infer that either $\lambda(\varepsilon) \equiv \lambda_{0}$ or else there is an integer $k \geq 1$ such that

$$
\lambda(\varepsilon)=\lambda_{0}+\varepsilon^{2 k} \lambda_{k}+O\left(\varepsilon^{2 k+2}\right) \quad \lambda_{k} \in \mathbb{R}-\{0\} .
$$

As a result, by Proposition 3.2 and (ㅍ1) we deduce, in particular,

$$
\boldsymbol{u}(\lambda)-\boldsymbol{u}_{0}=\varepsilon^{2} \boldsymbol{U},\|\boldsymbol{U}\|_{X^{2, q}} \leq M,
$$


with $M$ independent of $\varepsilon \rightarrow 0$. Likewise, from the analyticity properties of $\mathrm{w}$ and $\mathrm{v}$ and (4.13) we have

$$
\mathrm{W}-\boldsymbol{v}_{1}=\varepsilon \mathrm{W}, \quad \mathrm{v}=\varepsilon \mathrm{V}, \quad\|\mathrm{V}\|_{X^{2, q}}+\|\mathrm{W}\|_{\mathscr{W}_{2 \pi, 0}^{2}}^{2} \leq M
$$

Thus, since

$$
\boldsymbol{V}=\boldsymbol{u}_{0}+\varepsilon \boldsymbol{v}_{1}+\varepsilon\left[\left(\mathrm{w}(\varepsilon)-\boldsymbol{v}_{1}\right)+\mathrm{v}(\varepsilon)\right]+\boldsymbol{u}(\lambda)-\boldsymbol{u}_{0},
$$

(4.14) is a consequence of this identity and (4.25)-(4.28). We shall next prove the uniqueness property in (b) by adapting to the present case the abstract argument of [39, Theorem 8.B]. Let $\boldsymbol{z}=\overline{\boldsymbol{z}}+\boldsymbol{q}, \boldsymbol{q}:=\boldsymbol{z}-\overline{\boldsymbol{z}}$ be a $2 \pi$ periodic function where $\overline{\boldsymbol{z}} \in X_{0}^{2, q}(\Omega)$ and $\boldsymbol{q} \in \mathscr{W}_{2 \pi, 0}^{2}(\Omega)$ satisfy the first and the second equation in (4.8), respectively, with $\omega \equiv \widetilde{\omega}$ and $\lambda \equiv \widetilde{\lambda}$. By the uniqueness property associated with the implicit function theorem, the proof of the claimed uniqueness amounts to show that we can find a sufficiently small $\rho>0$ such that if

$$
\|\overline{\boldsymbol{z}}\|_{X^{2, q}}+\|\boldsymbol{q}\|_{\mathscr{W}_{2 \pi, 0}^{2}}+\left|\widetilde{\omega}-\omega_{0}\right|+\left|\widetilde{\lambda}-\lambda_{0}\right|<\rho,
$$

then there exists a neighborhood of $0, \mathcal{I}(0) \subset \mathbb{R}$, such that

$$
\begin{gathered}
\boldsymbol{q}=\eta \boldsymbol{v}_{1}+\eta \mathrm{y}, \overline{\boldsymbol{z}}=\eta \mathrm{z}, \text { for all } \eta \in \mathcal{I}(0), \\
\left|\widetilde{\omega}-\omega_{0}\right|+\left|\widetilde{\lambda}-\lambda_{0}\right|+\|\mathrm{z}\|_{X^{2, q}}+\|\mathrm{y}\|_{\mathscr{W}_{2 \pi, 0}^{2}} \rightarrow 0 \text { as } \eta \rightarrow 0 .
\end{gathered}
$$

To this end, we notice that, by (4.24), we may write

$$
\boldsymbol{q}=\mathbf{v}+\boldsymbol{y}
$$

where $\mathbf{v}=\left(\boldsymbol{y} \mid \boldsymbol{v}_{1}^{*}\right) \boldsymbol{v}_{1}+\left(\boldsymbol{y} \mid \boldsymbol{v}_{2}^{*}\right) \boldsymbol{v}_{2}$ and

$$
\left(\boldsymbol{y} \mid \boldsymbol{v}_{i}^{*}\right)=0, \quad i=1,2 .
$$

We next make the simple but important observation that if we modify $\boldsymbol{q}$ by a constant phase shift in time, $\delta$, namely, $\boldsymbol{q}(\tau) \rightarrow \boldsymbol{q}(\tau+\delta)$ it follows that the shifted function is still a $2 \pi$-periodic solution to (4.8) 2 and, moreover, by an appropriate choice of $\delta$,

$$
\mathbf{v}=\eta \boldsymbol{v}_{1},
$$

with $\eta=\eta(\delta) \in \mathbb{R}$. (The proof of (4.33) is straightforward, once we take into account (3.27).) Notice that from (4.29), (4.31)-(4.33) it follows that

$$
\begin{aligned}
|\eta| & \leq c_{0} \rho, \quad c_{0}>0, \\
\|\boldsymbol{y}\|_{\mathscr{W}_{2 \pi, 0}^{2}}^{2} & \leq \rho_{1}, \quad \rho_{1} \rightarrow 0 \text { as } \rho \rightarrow 0 .
\end{aligned}
$$


From (4.8), (4.31), and (4.33), we thus infer

$$
\widetilde{\mathscr{L}_{0}}(\overline{\boldsymbol{z}})=\mathcal{N}_{1}\left(\widetilde{\lambda}, \overline{\boldsymbol{z}}, \eta \boldsymbol{v}_{1}+\boldsymbol{y}\right)
$$

and

$$
\mathscr{Q}(\boldsymbol{y})=\mathscr{F}\left(\eta\left(\widetilde{\omega}-\omega_{0}\right), \eta\left(\widetilde{\lambda}-\lambda_{0}\right), \boldsymbol{v}_{1}\right)+\mathcal{N}(\eta, \widetilde{\lambda}, \widetilde{\omega}, \overline{\boldsymbol{z}}, \boldsymbol{y})
$$

In (4.35) the quantities $\mathcal{N}_{1}$ and $\mathscr{F}$ are defined in (4.8) 1 and (4.20), respectively, whereas

$$
\begin{aligned}
\mathcal{N}:= & \mathrm{P}\left\{-\eta\left(\widetilde{\lambda}-\lambda_{0}\right)\left[\left(\boldsymbol{u}(\widetilde{\lambda})-\boldsymbol{u}_{0}\right) \cdot \nabla \boldsymbol{v}_{1}+\boldsymbol{v}_{1} \cdot \nabla\left(\boldsymbol{u}(\widetilde{\lambda})-\boldsymbol{u}_{0}\right)\right]\right. \\
& -\eta \lambda_{0}\left[\left(\boldsymbol{u}(\widetilde{\lambda})-\boldsymbol{u}_{0}-\left(\widetilde{\lambda}-\lambda_{0}\right) \boldsymbol{u}^{\prime}\left(\lambda_{0}\right)\right) \cdot \nabla \boldsymbol{v}_{1}\right. \\
& +\boldsymbol{v}_{1} \cdot \nabla\left(\boldsymbol{u}(\widetilde{\lambda})-\boldsymbol{u}_{0}-\left(\widetilde{\lambda}-\lambda_{0}\right) \boldsymbol{u}^{\prime}\left(\lambda_{0}\right)\right]+\eta \widetilde{\lambda}\left[\boldsymbol{v}_{1} \cdot \nabla \boldsymbol{y}+\boldsymbol{y} \cdot \nabla \boldsymbol{v}_{1}\right. \\
& \left.\left.+\boldsymbol{v}_{1} \cdot \nabla \overline{\boldsymbol{z}}+\overline{\boldsymbol{z}} \cdot \nabla \boldsymbol{v}_{1}+\eta \boldsymbol{v}_{1} \cdot \nabla \boldsymbol{v}_{1}-\left(\eta \overline{\boldsymbol{v}_{1} \cdot \nabla \boldsymbol{v}_{1}}+\overline{\boldsymbol{v}_{1} \cdot \nabla \boldsymbol{y}}+\overline{\boldsymbol{y} \cdot \nabla \boldsymbol{v}_{1}}\right)\right]\right\} \\
& +\mathcal{N}_{2}(\widetilde{\lambda}, \widetilde{\omega}, \overline{\boldsymbol{z}}, \boldsymbol{y}),
\end{aligned}
$$

with $\mathcal{N}_{2}$ given in (4.8). We now observe the following facts.

(1) By (H1) and Proposition 3.2,

$$
\begin{array}{r}
\left\|\boldsymbol{u}(\widetilde{\lambda})-\boldsymbol{u}\left(\lambda_{0}\right)\right\|_{X^{2, q}} \leq M\left|\widetilde{\lambda}-\lambda_{0}\right| \\
\left\|\boldsymbol{u}(\widetilde{\lambda})-\boldsymbol{u}_{0}-\left(\widetilde{\lambda}-\lambda_{0}\right) \boldsymbol{u}^{\prime}\left(\lambda_{0}\right)\right\|_{X^{2, q}} \leq M\left|\widetilde{\lambda}-\lambda_{0}\right|^{2}
\end{array}
$$

where $M$ is independent of $\left|\widetilde{\lambda}-\lambda_{0}\right| \rightarrow 0$.

(2) Since $\widetilde{\mathscr{L}_{0}}$ is Fredholm of index 0, again by (स1), it is boundedly invertible.

(3) By Lemma 4.1 and (1) we easily show that

$$
\begin{aligned}
& \left\|\mathcal{N}_{1}\left(\tilde{\lambda}, \overline{\boldsymbol{z}}, \eta \boldsymbol{v}_{1}+\boldsymbol{y}\right)\right\|_{H_{q}} \\
& \quad \leq c_{1}\left(\left|\widetilde{\lambda}-\lambda_{0}\right|\|\overline{\boldsymbol{z}}\|_{X^{2, q}}+\|\overline{\boldsymbol{z}}\|_{X^{2, q}}^{2}+\eta^{2}+|\eta|\|\boldsymbol{y}\|_{\mathscr{W}_{2 \pi, 0}^{2}}+\|\boldsymbol{y}\|_{\mathscr{W}_{2 \pi, 0}^{2}}^{2}\right)
\end{aligned}
$$

and

$$
\begin{aligned}
\left\|\mathcal{N}-\mathcal{N}_{2}(\widetilde{\lambda}, \widetilde{\omega}, \overline{\boldsymbol{z}}, \boldsymbol{y})\right\|_{\mathscr{H}_{2 \pi, 0}} & \\
& \leq c_{2}\left(|\eta|\left|\widetilde{\lambda}-\lambda_{0}\right|^{2}+|\eta|\|\overline{\boldsymbol{z}}\|_{X^{2, q}}+\eta^{2}+|\eta|\|\boldsymbol{y}\|_{\mathscr{W}_{2 \pi, 0}^{2}}\right)
\end{aligned}
$$


Likewise,

$$
\begin{aligned}
& \left\|\mathcal{N}_{2}(\widetilde{\lambda}, \widetilde{\omega}, \overline{\boldsymbol{z}}, \boldsymbol{y})\right\| \mathscr{H}_{2 \pi, 0} \\
& \quad \leq c_{3}\left(\left(\left|\widetilde{\omega}-\omega_{0}\right|+\left|\widetilde{\lambda}-\lambda_{0}\right|\right)\|\boldsymbol{y}\|_{\mathscr{W}_{2 \pi, 0}}+\|\overline{\boldsymbol{z}}\|_{X^{2, q}}\|\boldsymbol{y}\|_{\mathscr{W}_{2 \pi, 0}^{2}}+\|\boldsymbol{y}\|_{\mathscr{W}_{2 \pi, 0}^{2}}^{2}\right)
\end{aligned}
$$

(4) By Proposition 3.5 and (4.22) we infer

$$
\begin{array}{r}
\eta\left(\widetilde{\lambda}-\lambda_{0}\right) \Re\left[\mu^{\prime}\left(\lambda_{0}\right)\right]=-\left(\mathcal{N} \mid \boldsymbol{v}_{1}^{*}\right) \\
-\eta\left(\widetilde{\omega}-\omega_{0}\right) \pi+\eta\left(\widetilde{\lambda}-\lambda_{0}\right)\left(\mathscr{F} \mid \boldsymbol{v}_{2}^{*}\right)=-\left(\mathcal{N} \mid \boldsymbol{v}_{2}^{*}\right),
\end{array}
$$

where the quantity

$$
\mathscr{F}=\mathscr{F}\left(\eta\left(\widetilde{\omega}-\omega_{0}\right), \eta\left(\widetilde{\lambda}-\lambda_{0}\right), \boldsymbol{v}_{1}\right),
$$

defined in (4.20), satisfies

$$
\left\|\mathscr{F}\left(\eta\left(\widetilde{\omega}-\omega_{0}\right), \eta\left(\widetilde{\lambda}-\lambda_{0}\right), \boldsymbol{v}_{1}\right)\right\|_{\mathscr{H}_{2 \pi, 0}} \leq c_{4}|\eta|\left(\left|\widetilde{\lambda}-\lambda_{0}\right|+\left|\widetilde{\omega}-\omega_{0}\right|\right) .
$$

(5) Proposition 3.5, (4.32), and (4.36) imply that

$$
\|\boldsymbol{y}\|_{\mathscr{W}_{2 \pi, 0}^{2}} \leq c_{5}\left(\left\|\mathscr{F}\left(\eta\left(\widetilde{\omega}-\omega_{0}\right), \eta\left(\widetilde{\lambda}-\lambda_{0}\right), \boldsymbol{v}_{1}\right)+\mathcal{N}(\eta, \widetilde{\lambda}, \widetilde{\omega}, \bar{z}, \boldsymbol{y})\right\|_{\mathscr{H}_{2 \pi, 0}}\right)
$$

With all the properties in (1)-(5) being established, we may now draw the following consequences. In the first place, by choosing $\rho$ sufficiently small and employing (4.34), from (4.35) and (4.36) we deduce

$$
\|\overline{\boldsymbol{z}}\|_{X^{2, q}}+\|\boldsymbol{y}\|_{\mathscr{W}_{2 \pi, 0}^{2}} \leq c_{6}\left(\eta^{2}+|\eta|\left(\left|\widetilde{\lambda}-\lambda_{0}\right|+\left|\widetilde{\omega}-\omega_{0}\right|\right)\right) .
$$

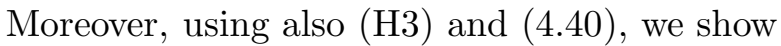

$$
\begin{aligned}
|\eta|\left(\left|\widetilde{\lambda}-\lambda_{0}\right|+\mid \widetilde{\omega}-\right. & \left.\omega_{0} \mid\right) \leq c_{7}\left[|\eta|\left(\mid \overline{\boldsymbol{z}}\left\|_{X^{2, q}}+\right\| \boldsymbol{y} \|_{\mathscr{W}_{2 \pi, 0}^{2}}\right)+\eta^{2}\right. \\
& \left.+\left(\left|\widetilde{\omega}-\omega_{0}\right|+\left|\widetilde{\lambda}-\lambda_{0}\right|\right)\|\boldsymbol{y}\|_{\mathscr{W}_{2 \pi, 0}^{2}}+\|\overline{\boldsymbol{z}}\|_{X^{2, q}}^{2}+\|\boldsymbol{y}\|_{\mathscr{W}_{2 \pi, 0}^{2}}^{2}\right] .
\end{aligned}
$$

Thus, combining (4.41) and (4.42), and taking $\rho$ sufficiently small we obtain, again with the help of (4.34),

$$
\|\overline{\boldsymbol{z}}\|_{X^{2, q}}+\|\boldsymbol{y}\|_{\mathscr{W}_{2 \pi, 0}^{2}} \leq c_{8}|\eta|^{2}
$$

which, once used back into (4.42), gives also

$$
\left|\widetilde{\lambda}-\lambda_{0}\right|+\left|\widetilde{\omega}-\omega_{0}\right| \leq c_{9}|\eta| .
$$


Recalling (4.31) and (4.33), by virtue of (4.43) and (4.44) we may establish the validity of (4.30), thus concluding the proof of the uniqueness property (b). It remains to show the statement in (c). To this end, we observe that if $\boldsymbol{v}(t):=\overline{\boldsymbol{v}}+\boldsymbol{w}$ is a solution to (4.8) in the function class specified in part (a), so is $\boldsymbol{v}^{\prime}:=\boldsymbol{v}(t+\pi)$. Let $\boldsymbol{w}^{\prime}:=\boldsymbol{v}^{\prime}-\overline{\boldsymbol{v}}^{\prime}$. By the uniqueness property of part (b), we must have (with the obvious meaning of the symbols) $\omega^{\prime}(\varepsilon)=\omega(\varepsilon)$ and $\lambda^{\prime}(\varepsilon)=\lambda(\varepsilon)$, for all $\varepsilon$ in a neighborhood of 0. However, if $\left(\boldsymbol{w} \mid \boldsymbol{v}_{1}^{*}\right)=\varepsilon \boldsymbol{v}_{1}$, then $\left(\boldsymbol{w}^{\prime} \mid \boldsymbol{v}_{1}^{*}\right)=-\varepsilon \boldsymbol{v}_{1}$. from which the stated parity condition follows. Finally, if $\lambda \not \equiv 0$, the expansion (4.26) must hold, and this implies $\lambda(\varepsilon)<\lambda_{0}$ or $\lambda(\varepsilon)>\lambda_{0}$, according to whether $\lambda_{k}$ (the first nonzero coefficient in the Taylor expansion for $\lambda$ ) is negative or positive. The theorem is completely proved.

Remark 4.2 As is well known, condition (플 $)$ means that when the Reynolds number $\lambda$ passes through the "critical" value $\lambda_{0}$ the eigenvalues of $\mathscr{L}(\lambda)$ must cross the imaginary axis at $\pm \mathrm{i} \omega_{0}$ with non-zero speed.

\section{$5 \quad$ Further Properties of Bifurcating Solutions}

The results of Theorem 4.1 ensure the existence and uniqueness of bifurcating time-periodic solutions in a neighborhood, $\mathscr{I}$, of $\left(\lambda_{0} ;\left(\boldsymbol{u}_{0}, p_{0}\right)\right)$, with period $2 \pi / \omega$ and $\omega$ "sufficiently close" to the imaginary part, $\omega_{0}$ of a (simple) purely imaginary eigenvalue of the relevant linearized operator $\mathscr{L}_{0}$. These solutions are of particular physical interest in that they may branch out only sub- or super-critically. However, the theorem cannot exclude the existence of other bifurcating time-periodic solutions in the same neighborhood $\mathscr{I}$, but with frequency not "close" to $\omega_{0}$, and having, in principle, different branching properties. Objective of this section is to prove that, under suitable further assumptions, the solutions determined in Theorem 4.1 are, in fact, the only possible bifurcating time-periodic solutions in $\mathscr{I}$. Roughly

speaking, these assumptions amount to say that as $\lambda$ passes $\lambda_{0}$, there are two and only two (complex conjugate) eigenvalues of the operator $\mathscr{L}_{0}$ crossing the imaginary axis, and that, in addition, for any nontrivial sequence $\left\{\boldsymbol{v}_{n}, \lambda_{n}, \omega_{n}\right\}$ of solutions to (4.1) with

$$
\left|\overline{\boldsymbol{v}}_{n}\left\|_{X^{2, q}}+\right\| \boldsymbol{w}_{n} \|_{\mathscr{W}_{2 \pi, 0}^{2}}+\right| \lambda_{n}-\lambda_{0} \mid \rightarrow 0
$$

there exists $\delta>0$ (which may depend on the sequence) such that

$$
\omega_{n} \geq \delta, \text { for all } n \in \mathbb{N} .
$$


From the physical viewpoint, this means that time-periodic bifurcating solutions branch out with a finite (nonzero) frequency. Numerical tests confirm that the above assumptions are indeed satisfied [17, Section 6],[9].

In order to prove the uniqueness result previously described, we need to show several preparatory lemmas, along the ideas developed in [22]. For $\omega>0$, let

$$
\mathscr{J}_{\omega}:=\int_{0}^{2 \pi}\left(\omega^{2}\left\|\partial_{\tau} \boldsymbol{w}(\tau)\right\|_{2}^{2}+\|\mathrm{P} \Delta \boldsymbol{w}(\tau)\|_{2}^{2}\right) d \tau
$$

Lemma 5.1 Let $\boldsymbol{w} \in \mathscr{W}_{2 \pi, 0}^{2}(\Omega)$. Then there is $c=c(\Omega)>0$ such that

$$
\begin{aligned}
& \int_{0}^{2 \pi}\|\boldsymbol{w}(\tau)\|_{r}^{2} d \tau \leq c \frac{1}{\omega^{\frac{2+r}{r}}} \mathscr{J}_{\omega}, \quad \text { all } r \geq 2, \\
& \max _{\tau \in[0,2 \pi]}\|\nabla \boldsymbol{w}(\tau)\|_{2}^{2} \leq \frac{1}{\omega} \mathscr{J}_{\omega} \\
& \int_{0}^{2 \pi}\|\boldsymbol{w}(\tau)\|_{4}^{4} d \tau \leq c \frac{1}{\omega^{3}} \mathscr{J}_{\omega}^{2} .
\end{aligned}
$$

Proof. We begin to observe that since $\overline{\boldsymbol{w}}(x)=\mathbf{0}$ for a.a. $x \in \Omega$, by the Wirtinger inequality we have

$$
\int_{0}^{2 \pi}|\boldsymbol{w}(x, \tau)|^{2} d \tau \leq \int_{0}^{2 \pi}\left|\partial_{\tau} \boldsymbol{w}(x, \tau)\right|^{2} d \tau
$$

which, in turn, after integration over $\Omega$ and using Fubini's theorem implies

$$
\int_{0}^{2 \pi}\|\boldsymbol{w}(\tau)\|_{2}^{2} d \tau \leq \frac{1}{\omega^{2}} \mathscr{J}_{\omega}
$$

Next, we notice that from the obvious identity

$$
\|\nabla \boldsymbol{w}\|_{2}^{2}=-\langle\mathrm{P} \Delta \boldsymbol{w}, \boldsymbol{w}\rangle
$$

and the Schwartz inequality it follows that

$$
\int_{0}^{2 \pi}\|\nabla \boldsymbol{w}(\tau)\|_{2}^{2} d \tau \leq\left(\int_{0}^{2 \pi}\|\mathrm{P} \Delta \boldsymbol{w}(\tau)\|_{2}^{2} d \tau\right)^{\frac{1}{2}}\left(\int_{0}^{2 \pi}\|\boldsymbol{w}(\tau)\|_{2}^{2} d \tau\right)^{\frac{1}{2}}
$$

which in conjunction with (5.2), by the Cauchy-Schwartz inequality delivers

$$
\int_{0}^{2 \pi}\|\nabla \boldsymbol{w}(\tau)\|_{2}^{2} d \tau \leq \frac{1}{2 \omega} \mathscr{J}_{\omega}
$$


Now, by classical interpolation, we have $\mathscr{W}_{2 \pi, 0}^{2}(\Omega) \subset C\left([0,2 \pi] ; W^{1,2}(\Omega)\right)$ and, for all $0 \leq s \leq \tau \leq 2 \pi$,

$$
\|\nabla \boldsymbol{w}(\tau)\|_{2}^{2}-\|\nabla \boldsymbol{w}(s)\|_{2}^{2}=\int_{s}^{\tau}\left\langle\partial_{\xi} \boldsymbol{w}(\xi), \mathrm{P} \Delta \boldsymbol{w}(\xi)\right\rangle d \xi
$$

see [30, Chapter 3.1]. Applying first the Schwartz inequality on the righthand side of this equation and then integrating over $s \in[0,2 \pi]$ we find for all $\tau \in[0,2 \pi]$

$\|\nabla \boldsymbol{w}(\tau)\|_{2}^{2} \leq \int_{0}^{2 \pi}\|\nabla \boldsymbol{w}(\xi)\|_{2}^{2} d \xi+\left(\int_{0}^{2 \pi}\left\|\partial_{\xi} \boldsymbol{w}(\xi)\right\|_{2}^{2} d \xi\right)^{\frac{1}{2}}\left(\int_{0}^{2 \pi}\|\mathrm{P} \Delta \boldsymbol{w}(\xi)\|_{2}^{2} d \xi\right)^{\frac{1}{2}}$.

Inequality (5.1) 2 is then a consequence of the latter and (5.3). Furthermore, we recall the well-known embedding inequality (see, e.g., [12, Lemma II.3.1])

$$
\|\boldsymbol{w}\|_{r} \leq c_{1}\|\boldsymbol{w}\|_{2}^{1-\lambda}\|\nabla \boldsymbol{w}\|_{2}^{\lambda}, \quad \lambda:=\frac{r-2}{r}, \quad r \geq 2 .
$$

Squaring both sides of the latter, integrating over $[0,2 \pi]$ and using Hölder inequality, we get

$$
\int_{0}^{2 \pi}\|\boldsymbol{w}(\tau)\|_{r}^{2} d \tau \leq c_{2}\left(\int_{0}^{2 \pi}\|\boldsymbol{w}(\tau)\|_{2}^{2} d \tau\right)^{1-\lambda}\left(\int_{0}^{2 \pi}\|\nabla \boldsymbol{w}(\tau)\|_{2}^{2} d \tau\right)^{\lambda}
$$

which, by virtue of (5.2) and (5.3) implies (5.1) 1 . Finally, choosing $r=4$ in (5.5) raising both sides to the power 4 and integrating over $[0,2 \pi]$ we show

$$
\int_{0}^{2 \pi}\|\boldsymbol{w}(\tau)\|_{4}^{4} d \tau \leq c_{3} \max _{\tau \in[0,2 \pi]}\|\boldsymbol{w}(\tau)\|_{2}^{2} \int_{0}^{2 \pi}\|\boldsymbol{w}(\tau)\|_{2}^{2} d \tau
$$

which with the help of (5.1) $)_{1,2}$ furnishes $(5.1)_{3}$.

Lemma 5.2 Let $\omega>0$, and let $\boldsymbol{v}$ be a $2 \pi$-periodic solution to (4.1) with $\overline{\boldsymbol{v}} \in X_{0}^{2 q}(\Omega)$ and $\boldsymbol{w}:=\boldsymbol{v}-\overline{\boldsymbol{v}} \in \mathscr{W}_{2 \pi, 0}^{2}(\Omega)$. Suppose that

$$
\|\overline{\boldsymbol{v}}\|_{X^{2 q}}+\|\boldsymbol{w}\|_{\mathscr{W}_{2 \pi, 0}^{2}} \leq \rho
$$

for some $\rho>0$. Then

$$
\omega \leq \sqrt{2 A+A^{2}}
$$

where $A:=\lambda^{2}\left(C_{1}+C_{2}\left(\rho+\rho^{2}\right)\right)$, with $C_{1}=C_{1}\left(\Omega,\|\boldsymbol{u}\|_{X^{2, q}}\right)>0$ and $C_{2}=$ $C_{2}(\Omega)>0$. 
Proof. From (4.1) we deduce that $\boldsymbol{w}$ satisfies the following equation

$$
\begin{aligned}
\omega \partial_{\tau} \boldsymbol{w}-\mathrm{P} \Delta \boldsymbol{w}= & \lambda \mathrm{P}\left[\partial_{1} \boldsymbol{w}-\boldsymbol{u}(\lambda) \cdot \nabla \boldsymbol{w}-\boldsymbol{w} \cdot \nabla \boldsymbol{u}(\lambda)\right] \\
& \quad+\lambda \mathrm{P}[\boldsymbol{w} \cdot \nabla \overline{\boldsymbol{v}}+\overline{\boldsymbol{v}} \cdot \nabla \boldsymbol{w}+\boldsymbol{w} \cdot \nabla \boldsymbol{w}-\overline{\boldsymbol{w} \cdot \nabla \boldsymbol{w}}] \\
:= & \lambda\left(\boldsymbol{g}_{1}+\boldsymbol{g}_{2}\right) .
\end{aligned}
$$

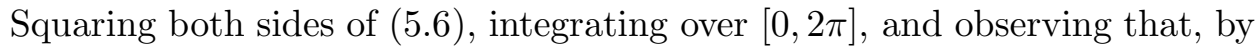
the $2 \pi$-periodicity and (5.4)

$$
\int_{0}^{2 \pi}\left\langle\partial_{\tau} \boldsymbol{w}, \Delta \boldsymbol{w}\right\rangle d \tau=0
$$

we infer

$$
\mathscr{J}_{\omega}=\lambda^{2} \int_{0}^{2 \pi}\left\|\boldsymbol{g}_{1}(\tau)+\boldsymbol{g}_{2}(\tau)\right\|_{2}^{2} d \tau .
$$

Arguing as in (4.7), for any $q \in(1,6 / 5)$ we show that

$$
\int_{0}^{2 \pi}\left\|\boldsymbol{g}_{1}(\tau)\right\|_{2}^{2} d \tau \leq c_{1} \int_{0}^{2 \pi}\left(\|\nabla \boldsymbol{w}(\tau)\|_{2}^{2}+\|\boldsymbol{w}(\tau)\|_{q^{\prime}}^{2}\right) d \tau
$$

where, $c_{1}=c_{1}\left(\Omega,\|\boldsymbol{u}\|_{X^{2, q}}\right)$. The latter inequality, in conjunction with (5.1) furnishes

$$
\int_{0}^{2 \pi}\left\|\boldsymbol{g}_{1}(\tau)\right\|_{2}^{2} d \tau \leq c_{2}\left(\frac{1}{\omega^{\frac{2+q^{\prime}}{q^{\prime}}}}+\frac{1}{\omega}\right) \mathscr{J}_{\omega}
$$

where, here and in the rest of the proof, $c_{i}, i=2, \ldots$, denotes a positive constant depending at most on $\Omega$. Likewise, we show

$$
\int_{0}^{2 \pi}\|\boldsymbol{w} \cdot \nabla \overline{\boldsymbol{v}}(\tau)+\overline{\boldsymbol{v}} \cdot \nabla \boldsymbol{w}(\tau)\|_{2}^{2} d \tau \leq c_{3} \rho^{2}\left(\frac{1}{\omega^{\frac{2+q^{\prime}}{q^{\prime}}}}+\frac{1}{\omega}\right) \mathscr{J}_{\omega} .
$$

Moreover

$$
\int_{0}^{2 \pi}\|\boldsymbol{w} \cdot \nabla \boldsymbol{w}(\tau)\|_{2}^{2} d \tau \leq\left(\int_{0}^{2 \pi}\|\boldsymbol{w}(\tau)\|_{4}^{4} d \tau\right)^{\frac{1}{2}}\left(\int_{0}^{2 \pi}\|\nabla \boldsymbol{w}(\tau)\|_{4}^{4} d \tau\right)^{\frac{1}{2}}
$$

so that by (4.6), (5.1) 3 and by assumption we conclude

$$
\int_{0}^{2 \pi}\|\boldsymbol{w} \cdot \nabla \boldsymbol{w}(\tau)\|_{2}^{2} d \tau \leq c_{4} \rho \frac{1}{\omega^{\frac{3}{2}}} \mathscr{J}_{\omega} .
$$


Finally, we observe that

$$
\int_{0}^{2 \pi}\|\overline{\boldsymbol{w} \cdot \nabla \boldsymbol{w}}\|_{2}^{2} d \tau \leq 2 \pi \int_{0}^{2 \pi}\|\boldsymbol{w} \cdot \nabla \boldsymbol{w}(\tau)\|_{2}^{2} d \tau
$$

so that by (5.10) it follows

$$
\int_{0}^{2 \pi}\|\overline{\boldsymbol{w} \cdot \nabla \boldsymbol{w}}\|_{2}^{2} d \tau \leq c_{5} \rho \frac{1}{\omega^{\frac{3}{2}}} \mathscr{J}_{\omega} .
$$

Collecting (5.8)-(5.11) and observing that, by Young's inequality,

$$
\frac{1}{\omega^{\frac{2+q^{\prime}}{q^{\prime}}}}=\frac{1}{\omega^{\frac{4}{q^{\prime}}}} \frac{1}{\omega^{\frac{q^{\prime}-2}{q^{\prime}}}} \leq c(q)\left(\frac{1}{\omega^{2}}+\frac{1}{\omega}\right), \quad \frac{1}{\omega^{\frac{3}{2}}}=\frac{1}{\omega} \frac{1}{\sqrt{\omega}} \leq \frac{1}{2}\left(\frac{1}{\omega^{2}}+\frac{1}{\omega}\right),
$$

we find

$$
\int_{0}^{2 \pi}\left\|\boldsymbol{g}_{1}(\tau)+\boldsymbol{g}_{2}(\tau)\right\|_{2}^{2} \leq\left(C_{1}+C_{7}\left(\rho+\rho^{2}\right)\right)\left(\frac{1}{\omega^{2}}+\frac{1}{\omega}\right) \mathscr{J}_{\omega} .
$$

Combining this latter inequality with (5.7), we get

$$
\frac{1}{\omega^{2}}+\frac{1}{\omega} \geq \frac{1}{A},
$$

where $A$ is the quantity defined in the statement of the lemma. The proof is then accomplished by employing in (5.12) the elementary inequality

$$
\frac{1}{\omega} \leq \frac{1}{2 A}+\frac{A^{2}}{2 \omega^{2}}
$$

Lemma 5.3 Let $\omega_{*}>0$ and let

$$
\mathscr{Q}_{*}: \boldsymbol{w} \in \mathscr{W}_{2 \pi, 0}^{2}(\Omega) \mapsto \omega_{*} \partial_{\tau} \boldsymbol{w}-\mathscr{L}_{0}(\boldsymbol{w}) \in \mathscr{H}_{2 \pi, 0}(\Omega) .
$$

Then, $\mathscr{Q}_{*}$ is boundedly invertible if and only if $\mu_{* k}:=\mathrm{i} k \omega_{*} \notin \sigma\left(\mathscr{L}_{0}\right)$ for all $k \in \mathbb{N}-\{0\}$.

Proof. From Proposition 3.3 we know that the $\mu_{* k}$ 's can only be eigenvalues of $\mathscr{L}_{0}$. (10) Since, by assumption, $\mathrm{N}\left[\mathscr{L}_{0} \pm \mu_{* k} I\right]=\{0\}$, for all $k$, with the help of Lemma 3.3 we deduce that the operator

$$
\left(\mathscr{L}_{0} \pm \mu_{* k} I\right)^{-1}
$$

\footnotetext{
${ }^{(10)}$ In fact, the same proposition guarantees that there is at most a finite number of such $\mu_{* k}$ 's.
} 
is a homeomorphism of $H_{\mathbb{C}}(\Omega)$ onto $Z_{\mathbb{C}}^{2,2}(\Omega)$. Therefore, by using classical Fourier series techniques, we show that $\mathscr{Q}_{*}$ is also boundedly invertible with

$$
\mathscr{Q}_{*}^{-1}: \boldsymbol{f} \in \mathscr{H}_{2 \pi, 0}(\Omega) \mapsto \boldsymbol{w} \in \mathscr{W}_{2 \pi, 0}^{2}(\Omega),
$$

where

$$
\boldsymbol{w}(t):=\sum_{\ell=-\infty, \ell \neq 0}^{\infty} \mathrm{e}^{\mathrm{i} \ell \tau}\left(\mathscr{L}_{0}-\mathrm{i} \ell \omega_{*} I\right)^{-1}\left[\frac{1}{2 \pi} \int_{0}^{2 \pi} \boldsymbol{f}(s) \mathrm{e}^{-\mathrm{i} \ell s} d s\right]
$$

We are now in a position to prove the main result of this section.

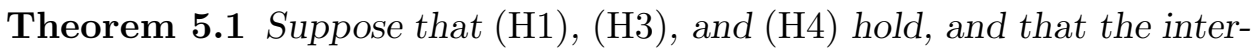
section of the spectrum of the operator $\mathscr{L}_{0}$ with the imaginary axis consists of two and only two (complex conjugate) simple eigenvalues $\pm \mathrm{i} \omega_{0}$. Then, there exists $\rho>0$ such that every non-trivial $2 \pi$-periodic solution $\boldsymbol{v}$ to (4.1) for some $\omega>0$, for which

$$
\|\overline{\boldsymbol{v}}\|_{X^{2, q}}+\|\boldsymbol{w}\|_{\mathscr{W}_{2 \pi, 0}^{2}}+\left|\lambda-\lambda_{0}\right|<\rho
$$

must belong (up to a phase shift) to the one-parameter family of solutions constructed in Theorem 4.1,

Proof. In view of the uniqueness result of Theorem 4.1(b), if the claim is not true, there should exist a (non-trivial) sequence of solutions to (4.1), $\left\{\boldsymbol{v}_{n}, \lambda_{n}, \omega_{n}\right\}$, and a number $a>0$ such that

$$
\left\|\overline{\boldsymbol{v}}_{n}\right\|_{X^{2, q}}+\left\|\boldsymbol{w}_{n}\right\|_{\mathscr{W}_{2 \pi, 0}^{2}}+\left|\lambda_{n}-\lambda_{0}\right| \rightarrow 0
$$

and

$$
\left|\omega_{n}-\omega_{0}\right| \geq a .
$$

By virtue of Lemma 5.2, the sequence $\left\{\omega_{n}\right\}$ is bounded, so that, by (H4), there exists $\omega_{*}>0$, such that

$$
\left|\omega_{n}-\omega_{*}\right| \rightarrow 0, \quad \omega_{*} \neq \omega_{0}
$$

From (4.1) we thus obtain that $\overline{\boldsymbol{v}}$ and $\boldsymbol{w}$ solve the following coupled equations

$$
\begin{aligned}
& \widetilde{\mathscr{L}}(\overline{\boldsymbol{v}})=\mathcal{N}_{1}\left(\lambda_{0}+\sigma, \overline{\boldsymbol{v}}, \boldsymbol{w}\right) \text { in } H_{q}(\Omega), \\
& \mathscr{Q}_{*}(\boldsymbol{w})=\mathcal{N}_{* 2}\left(\lambda_{0}+\sigma, \omega_{*}+\xi, \overline{\boldsymbol{v}}, \boldsymbol{w}\right) \text { in } \mathscr{H}_{2 \pi, 0}^{2}(\Omega),
\end{aligned}
$$


where $\sigma:=\lambda-\lambda_{0}, \xi:=\omega-\omega_{*}, \mathcal{N}_{1}$ and $\mathscr{Q}_{*}$ are defined in (4.8) 1 and (5.14), respectively, whereas $\mathcal{N}_{* 2}$ is given in (4.8) 2 with $\omega_{0} \equiv \omega_{*}$. Consider the map

$$
\begin{gathered}
\mathcal{M}:(\sigma, \xi, \overline{\boldsymbol{v}}, \boldsymbol{w}) \in \mathcal{I}(0) \times U(0) \times X_{0}^{2, q}(\Omega) \times \mathscr{W}_{2 \pi, 0}^{2}(\Omega) \\
\mapsto\left(\widetilde{\mathscr{L}_{0}}(\overline{\boldsymbol{v}})-\mathcal{N}_{1}\left(\lambda_{0}+\sigma, \overline{\boldsymbol{v}}, \boldsymbol{w}\right), \mathscr{Q}_{*}(\mathrm{w})-\mathcal{N}_{* 2}\left(\lambda_{0}+\sigma, \omega_{*}+\xi, \overline{\boldsymbol{v}}, \boldsymbol{w}\right)\right) \\
\in H_{q}(\Omega) \times \mathscr{H}_{2 \pi, 0}(\Omega) .
\end{gathered}
$$

Clearly, the equation $\mathcal{M}(0,0, \overline{\boldsymbol{v}}, \boldsymbol{w})=\mathbf{0}$ has the solution $\mathrm{U}_{0}:=(\overline{\boldsymbol{v}}=\mathbf{0}, \boldsymbol{w}=$ $\mathbf{0})$. The Fréchet derivative, $D \mathcal{M}$, of $\mathcal{M}$ with respect to $(\overline{\boldsymbol{v}}, \boldsymbol{w})$ evaluated at $\left(\sigma=0, \xi=0, \mathrm{U}_{0}\right)$ is given by

$D \mathcal{M}:(\mathrm{v}, \mathrm{w}) \in X_{0}^{2, q}(\Omega) \times \mathscr{W}_{2 \pi, 0}^{2}(\Omega) \mapsto\left(\widetilde{\mathscr{L}}_{0}(\mathrm{v}), \mathscr{Q}_{*}(\mathrm{w})\right) \in H_{q}(\Omega) \times \mathscr{H}_{2 \pi, 0}(\Omega)$,

which, by (H1) and Lemma 5.3 is a bijection. Therefore, by the implicit function theorem, there are no nontrivial solutions satisfying (5.15) and (5.16), thus showing a contradiction.

\section{References}

[1] Andronov, A.A., and Witt, A. Sur la theórie mathematiques des autooscillations, C. R. Acad. Sci. Paris, 190 (1930) 256-258

[2] Babenko, K. I., The stationary solutions of the problem of the flow around a body by a viscous incompressible fluid, Math. USSR-Sb., 20 $1-25(1973)$

[3] Babenko, K.I., On properties of steady viscous incompressible fluid flows. Approximation methods for Navier-Stokes problems (Proc. Sympos., Univ. Paderborn, Paderborn, 1979), pp. 12-42, Lecture Notes in Math., Vol. 771, Springer, Berlin, 1980

[4] Babenko, K. I., On the spectrum of a linearized problem on the flow of a viscous incompressible fluid around a body (Russian), Dokl. Akad. Nauk SSSR, 262 64-68 (1982)

[5] Babenko, K.I., Periodic solutions of a problem of the flow of a viscous fluid around a body, Soviet Math. Dokl. 25 211-216 (1982)

[6] Chossat, P., and Iooss, G., The Couette-Taylor problem, Applied Mathematical Sciences, Vol. 102, Springer-Verlag, New York (1994) 
[7] Crandall, M.G., and Rabinowitz, P.H., The Hopf bifurcation theorem in infinite dimensions, Arch. Rational Mech. Anal., 67 53-72 (1977)

[8] Farwig, R., and Neustupa, J., Spectral properties in $L^{q}$ of an Oseen operator modelling fluid flow past a rotating body, Tohoku Math. J. 62 287-309 (2010)

[9] Fatone, L., Gervasio, P., and Quarteroni, A., Multimodels for incompressible flows, J. Math. Fluid Mech. 2 126-150 (2000)

[10] Galdi, G.P., On the Oseen boundary value problem in exterior domains. The Navier-Stokes equations II -theory and numerical methods (Oberwolfach, 1991), 111-131, Lecture Notes in Math., 1530, Springer, Berlin (1992)

[11] Galdi, G.P., Stationary Navier-Stokes problem in a two-dimensional exterior domain, Stationary partial differential equations. Vol. I, 71155, Handb. Differ. Equ., North-Holland, Amsterdam (2004)

[12] Galdi, G.P., An introduction to the mathematical theory of the NavierStokes equations. Steady-state problems, Second edition. Springer Monographs in Mathematics, Springer, New York (2011)

[13] Galdi, G.P., Existence and uniqueness of time-periodic solutions to the Navier-Stokes equations in the whole plane, Discrete Contin. Dyn. Syst. Ser. S, 6 1237-1257 (2013)

[14] Galdi, G.P., On time-periodic flow of a viscous liquid past a moving cylinder, Arch. Ration. Mech. Anal., 210 451-498 (2013)

[15] Galdi, G.P., and Rabier, P.J., Sharp existence results for the stationary Navier-Stokes problem in three-dimensional exterior domains, Arch. Rational Mech. Anal., 154 343-368 (2000)

[16] Galdi, G.P., and Sohr, H., Existence and uniqueness of time-periodic physically reasonable Navier-Stokes flow past a body, Arch. Rational Mech. Anal. 172 363-406 (2004)

[17] Gerecht, D., Rannacher R., and Wollner, W., Computational aspects of pseudospectra in hydrodynamic stability analysis, J. Math. Fluid Mech., 14, 661-692 (2012)

[18] Gohberg, I., Goldberg, S. and Kaashoek, M.A., Classes of linear operators:I. Operator Theory, Advances and Applications, Vol.49, Birkhäuser Verlag, Basel (1990) 
[19] Hopf, E., Abzweigung einer periodischen Lösung von einer stationären eines Differentialsystems, Ber. Verh. Schs. Akad. Wiss. Leipzig. Math.Nat. Kl. 95, 3-22 (1943)

[20] Iooss, G., Existence et stabilité de la solution périodiques secondaire intervenant dans les problémes d'evolution du type Navier-Stokes, Arch. Rational Mech. Analysis, 47, 301-329 (1972)

[21] Iooss, G., and Joseph, D.D., Bifurcation and stability of $n T$-periodic solutions branching from $T$-periodic solutions at points of resonance, Arch. Rational. Mech. Anal., 66 135-172 (1977)

[22] Iudovich, V.I., The onset of auto-oscillations in a fluid, J. Appl. Math. Mech. 35, , 587-603 (1971)

[23] Joseph, D.D., and Sattinger, D.H., Bifurcating time periodic solutions and their stability. Arch. Rational Mech. Anal., 45, 79-109 (1972)

[24] Kang, K., Miura, H., and Tsai, T-P., Asymptotics of small exterior Navier-Stokes flows with non-decaying boundary data, Comm. Partial Differential Equations, 37 1717-1753 (2012)

[25] Kato, T., Perturbation theory for linear operators, Springer Classics in Mathematics (1995)

[26] Kielhöfer, H., Bifurcation theory. An introduction with applications to partial differential equations, Applied Mathematical Sciences, Vol. 156. Springer, New York, (2012)

[27] Kyed, M., The existence and regularity of time-periodic solutions to the three-dimensional Navier-Stokes equations in the whole space, Nonlinearity 27 2909-2935 (2014)

[28] Kozono, H., and Nakao, M., Periodic solutions of the Navier-Stokes equations in unbounded domains Tôhoku Math. J., 48 33-50 (1996)

[29] Lindtstedt, A., Beitrag zur Integration der Differentialgleichungen der Störungstheorie, Abh. K. Akad. Wiss. St. Petersburg, 31 (4) (1882)

[30] Lions, J.-L., and Magenes, E., Non-homogeneous boundary value problems and applications, Vol. 1, Springer-Verlag Berlin Heidelberg New York (1972) 
[31] Maremonti, P., Existence and stability of time periodic solutions of the Navier-Stokes equations in the whole space, Nonlinearity, 4 503-529 (1991)

[32] Maremonti, P., and Padula, M., Existence, uniqueness and attainability of periodic solutions of the Navier-Stokes equations in exterior domains, J. Math. Sci., 93 719-746 (1999)

[33] Melcher, A., Schneider, G., and Uecker, H., A Hopf-bifurcation theorem for the vorticity formulation of the Navier-Stokes equations in $\mathbb{R}^{3}$, Comm. Partial Differential Equations, 33 772-783 (2008)

[34] Nguyen, T.-H., Periodic motions of Stokes and Navier-Stokes flows around a rotating obstacle, Arch. Rational Mech. Anal. 213 689-703 (2014)

[35] Poincaré, H., Les Methodes Nouvelles de la Mecanique Celeste, Vol. I, Paris (1892)

[36] Sazonov, L.I., The onset of auto-oscillations in a flow, Siberian Math. J. 35 1202-1209 (1994)

[37] Tritton, D.J., Physical Fluid Dynamics, Second Edition, Clarendon Press, Oxford (1988)

[38] Yamazaki, M., The Navier-Stokes equations in the weak- $L^{n}$ space with time-dependent external force, Math. Ann., 317 635-675 (2000)

[39] Zeidler, E., Nonlinear Functional Analysis and Applications, Vol.1, Fixed-Point Theorems, Springer-Verlag, New York (1986)

[40] Zeidler, E., Nonlinear Functional Analysis and Applications, Vol.4, Application to Mathematical Physics, Springer-Verlag, New York (1988) 